\title{
Bioinformatic analysis of PLOD family member expression and prognostic value in non-small cell lung cancer
}

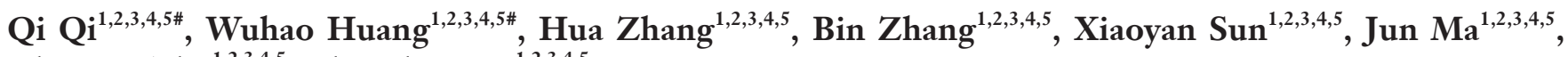 \\ Chaonan Zhu ${ }^{1,2,3,4,5}$, Changli Wang ${ }^{1,2,3,4,5}$
}

${ }^{1}$ Department of Lung Cancer, Tianjin Medical University Cancer Institute and Hospital, Tianjin, China; ${ }^{2}$ National Clinical Research Center for Cancer, Tianjin, China; ${ }^{3}$ Key Laboratory of Cancer Prevention and Therapy, Tianjin, China; ${ }^{4}$ Tianjin's Clinical Research Center for Cancer, Tianjin, China; ${ }^{5}$ Tianjin Lung Cancer Center, Tianjin, China

Contributions: (I) Conception and design: C Wang, Q Qi, W Huang; (II) Administrative support: None; (III) Provision of study materials: Q Qi, W Huang; (IV) Collection and assembly of data: H Zhang, X Sun; (V) Data analysis and interpretation: Q Qi, W Huang, J Ma, C Zhu; (VI) Manuscript writing: All authors; (VII) Final approval of manuscript: All authors.

\#These authors contributed equally to this work.

Correspondence to: Changli Wang, MD. Huanhuxi Road, Hexi District, Tianjin 300202, China. Email: wangchangli@tjmuch.com.

Background: Procollagen-lysine, 2-oxoglutarate 5-dioxygenases (PLODs) are a group of enzymes that can mediate the hydroxylation of lysyl to hydroxylysine and participate in the formation of stabilized collagen. Evidence has demonstrated that PLODs are involved in the steps of tumor progression, including proliferation, invasion, and metastasis. However, limited information is available on the function of PLOD1/2/3 in lung cancer. In this study, we investigated the expression patterns and prognostic values of PLODs in patients with lung adenocarcinoma (LUAD) and lung squamous cell carcinoma (LUSC).

Methods: The Oncomine database and UALCAN were used to analyze the mRNA expression levels of PLOD family members in non-small cell lung cancer (NSCLC). The prognostic values of PLODs were investigated by the Kaplan-Meier Plotter database. We collected 33 patients with lung cancer to further verify the expression profiles and prognostic values of PLODs. The Kaplan-Meier method was used to perform survival curves, and the log-rank test was performed to evaluate the differences in survival. According to the GSE31210 databset, univariate and multivariate analyses were performed to identify whether PLODs were independent prognostic indicators for survival. Meanwhile, we investigated the mutations, potential biological functions and immune relevance of PLODs on the basis of the cBioPortal, Metascape and TIMER databases respectively.

Results: We found that the mRNA and protein expression levels of PLODs in NSCLC tissues were higher than those in normal lung tissues. High PLOD1/2/3 expression had significant relevance to poor survival in LUAD but not in LUSC. In addition, the GSE31210 dataset showed that PLOD1 and PLOD3 were independent risk factors for relapse-free survival and overall survival (OS) in LUAD. We observed a high alteration rate of PLODs in LUSC patients, and the genetic alterations of PLODs had significant relevance to favorable OS. Furthermore, we observed that PLODs were significantly associated with tumor immunity in lung cancer. The enrichment analysis of the Kyoto Encyclopedia of Genes and Genomes (KEGG) pathway showed that the functions of the PLODs focused on cell cycle, DNA replication, and glycolysis/ gluconeogenesis in LUAD.

Conclusions: These results indicated that PLODs were highly expressed in lung cancer and may be suitable prognostic markers.

Keywords: Bioinformatic analysis; lung cancer; online database; procollagen-lysine; 2-oxoglutarate 5-dioxygenase (PLOD); prognosis 
Submitted Jan 12, 2021. Accepted for publication May 06, 2021.

doi: $10.21037 /$ tcr-21-73

View this article at: https://dx.doi.org/10.21037/tcr-21-73

\section{Introduction}

As the most common cause of cancer mortality worldwide, lung cancer causes approximately 2 million new cases and 1.8 million deaths each year (1). Based on histological characteristics, lung cancer is categorized into non-small cell lung cancer (NSCLC) and small-cell lung cancer. NSCLC is the major pathological type of lung cancer, and it is further categorized into lung squamous cell carcinoma (LUSC) and lung adenocarcinoma (LUAD). NSCLC is prone to relapse and metastasis, which generally leads to poor prognosis, as evidenced by a 5 -year survival rate of less than $20 \%$ (2). Despite advanced progress in various treatments, including surgery, medicine, and radiotherapy, the long-term survival of NSCLC patients has not evidently improved.

In recent years, the importance of the tumor microenvironment (TME) in the biological behavior of tumors has drawn increasing attention. As the main component of the TME, the extracellular matrix (ECM) plays a critical role in the development of tumors (3-5). The ECM is composed of multifarious components, and collagen is regarded as the most abundant protein. Increasing studies indicate that the interactions between collagen and cells may trigger biochemical and biophysical signals that influence normal biological function and cancer development $(6,7)$. The collagen precursor is synthesized in the rough endoplasmic reticulum and subsequently undergoes a series of processes and modifications, including lysine hydroxylation. As the critical procedure of collagen biosynthesis, the hydroxylation of lysyl residues usually occur at the $\mathrm{Y}$ position of the repeating Gly-X-Y motif and play a key role in the formation of collagen crosslinks and glycosylation $(8,9)$. Aberrant lysyl hydroxylation is correlated with the development of some collagen-related diseases, including cancer (10). The hydroxylation of lysyl to form hydroxylysine depends on prolyl 4-hydroxylation and procollagen-lysine,2-oxoglutarate 5-dioxygenase (PLOD). To date, three PLOD isoforms, PLOD1, PLOD2, and PLOD3, have been identified and share a high degree of homology in protein sequences (9). The PLOD1, PLOD2, and PLOD3 genes are located at chromosomes 1p36, 3q23$\mathrm{q} 24$, and $7 \mathrm{q} 36$, respectively $(9,11,12)$.

After the diagnosis of NSCLC, the prognosis needs to be considered. Although TNM staging could serve as the most classic prognostic indicator, other prognostic factors are also necessary to be identified. Several studies have shown that abnormal expression levels of some molecules, including CEA, CA125 and CA153, could be predictive for the survival of patients with lung cancer (13). Notably, abnormal expression levels of PLODs are observed in various cancers. The expression level of PLOD1 in gastrointestinal carcinoma was elevated, and high expression of PLOD1 was related to poor clinical prognosis (14). A previous study reported that the expression levels of PLOD family members in gastric cancer were upregulated and associated with shorter overall survival (OS), initial progression and post progression survival (15). Additionally, PLOD2 and PLOD3 have been shown to promote tumor progression and metastasis in lung cancer and glioma $(16,17)$. Therefore, PLOD family members could serve as potential prognostic biomarkers. The molecular characteristics that clarify the interaction between PLODs and NSCLC have yet to be elucidated. The investigation between PLOD family members and NSCLC will contribute to identifying the novel prognostic factors and potential molecular mechanisms which are related to the progression of NSCLC.

In this study, bioinformatic analyses of public databases were performed to investigate the differential expression, mutation, and prognostic value of PLOD family members. We analyzed the potential biological functions of PLODs and further investigated the correlations between PLODs and immune indicators, including immune-related molecules and immune-infiltrated cells. Our research showed that PLODs were highly expressed in NSCLC tissues compared to normal tissues. High expression levels of PLODs were significantly related to poor survival in patients with LUAD. This study may provide an initial basis for research on PLODs in lung cancer. We present the following article/case in accordance with the REMARK reporting checklist (available at https://dx.doi.org/10.21037/ tcr-21-73).

\section{Methods}

\section{Patients and specimens}

A total of 33 patients with NSCLC, including 19 with LUAD and 14 with LUSC, were included in this study. The 
tumor tissues and matched normal tissues were obtained during the operation. All patients underwent surgery at Tianjin Medical University Cancer Institute and Hospital between 2008 and 2011. Patients with radiotherapy and chemotherapy before surgery were excluded. All collected tumor tissues and normal lung tissues were stored at $-80{ }^{\circ} \mathrm{C}$ until required. The study was conducted in accordance with the Declaration of Helsinki (as revised in 2013). The study was approved by the institutional review board of Tianjin Medical University Cancer Institute and Hospital (NO.: bc2020193) and informed consent was taken from all the patients.

\section{Quantitative RT-qPCR}

Total RNA was extracted from lung cancer and normal tissues by TRIzol reagent and then qualified and quantified using a NanoDrop system. One microgram of RNA was transcribed to cDNA using a Prime-Script ${ }^{\mathrm{TM}}$ II 1st Strand cDNA Synthesis Kit. RT-qPCR was performed using a CFX96 Real-time System. The PCR conditions were as follows: $94{ }^{\circ} \mathrm{C}$ for $1 \mathrm{~min}, 55^{\circ} \mathrm{C}$ for $1.5 \mathrm{~min}$, and $72{ }^{\circ} \mathrm{C}$ for $5 \mathrm{~min}$, with a total of 30 cycles. GAPDH was used as the internal control, and qPCR was performed with the following primers:

PLOD1-Forward: AAGCCGGAGGACAACCTTTTA PLOD1-Reverse: GCGAAGAGAATGACCAGATCC PLOD2-Forward: CATGGACACAGGATAATGGCTG PLOD2-Reverse: AGGGGTTGGTTGCTCAATAAAAA PLOD3-Forward: AGAAGGTCCGGTGGTTAAAGA PLOD3-Reverse: CGGCCAGAATCACGTCGTAG GAPDH-Forward: GGAGCGAGATCCCTCCAAAAT GAPDH-Reverse: GGCTGTTGTCATACTTCTCATGG

\section{Oncomine analysis}

The Oncomine online cancer microarray database (www. oncomine.org) was used to analyze the mRNA expression levels of PLOD family members in various cancers. Based on Student's $t$-test, we analyzed the different mRNA expression levels of PLOD family genes between tumor and normal tissues. The parameters, including the $\mathrm{P}$-value, fold change, and gene rank, were established at $0.01,1.5$, and $10 \%$, respectively.

\section{UALCAN}

The interactive web portal UALCAN (http://ualcan.path. uab.edu) (18) was utilized to analyze the corresponding expression of the target gene in tumor and normal specimens on the basis of The Cancer Genome Atlas (TCGA) database. The corresponding expression of the gene of interest can be investigated in accordance with the different tumor subgroups (e.g., tumor stage, histological subtype, and sex).

\section{Human Protein Atlas}

The Human Protein Atlas website (https://www. proteinatlas.org) (19) contains abundant protein expression data based on the immunohistochemistry of multifarious tissues and is composed of the Tissue, Cell, and Pathology Atlases. In accordance with the immunohistochemistry images, the protein expression levels of PLOD1/2/3 were compared between normal and lung cancer tissues.

\section{Kaplan-Meier plotter}

The prognostic values of PLOD family members were investigated using the Kaplan-Meier Plotter online database (www.kmplot.com), which contains gene expression data and survival-related information of lung cancer (20). In accordance with the median mRNA expression, we divided patients with LUAD and LUSC into high- and low-expression groups. The differences in survival were compared using statistical parameters, including the hazard ratio, $95 \%$ confidence interval (CI), and log-rank $\mathrm{P}$ value. A $\mathrm{P}$ value $<0.05$ was considered statistically significant.

\section{cBioPortal}

As an open-access online resource, cBioPortal (www. cbioportal.org) can be utilized to analyze and visualize multidimensional cancer genomics data (21). In this study, the LUAD and LUSC datasets (TCGA, PanCancer Atlas) were used to analyze the genetic alterations in PLOD1, PLOD2, and PLOD3. The genomic profiles contained mutations, putative copy-number alterations, and mRNA expression (RNA Seq V2 RSEM with z-scores $= \pm 2$ ). Based on the Kaplan-Meier plot and the log-rank test, we investigated the correlations between the genetic alterations of PLODs and survival, including OS and disease-free survival (DFS), in patients with LUAD and LUSC. In addition, cBioPortal was applied to assess the correlations among the PLOD family genes and extract the co-expressed 
genes of PLODs in LUAD and LUSC.

\section{Metascape analysis}

Metascape (http://metascape.org) (22) was utilized to perform pathway and enrichment analyses of the PLOD $1 / 2 / 3$ and co-expressed genes extracted from cBioPortal. The co-expressed genes were considered to have good correlations with PLOD family members at a Spearman's correlation coefficient $\geq 0.4$. In accordance with Metascape, we carried out enrichment analyses, including Gene Ontology (GO) and Kyoto Encyclopedia of Genes and Genomes (KEGG) pathway analyses. GO analyses were composed of biological processes, cellular components, and molecular functions. A P-value $<0.05$, minimum count of 3 , and enrichment factor $>1.5$ were considered statistically significant.

\section{Immune-related analysis in the TIMER database}

The comprehensive website resource TIMER (http:// cistrome.shinyapps.io/timer) was applied to perform the systematic investigation of immune infiltrates across diverse cancers (23). We investigated the correlations between PLOD family members and tumor-infiltrating immune cells, including B cells, CD4+ T cells, CD8+ T cells, neutrophils, macrophages, and dendritic cells, in LUAD and LUSC. We also explored the relationships of PLOD expression with immune-related genes, including programmed cell death 1 ligand 1 [PD-L1 (CD274)], C-X-C motif chemokine ligand 10 (CXCL10), granzyme $\mathrm{A}(G Z M A)$, and granzyme $\mathrm{B}(G Z M B)$.

\section{Gene Expression Omnibus (GEO) database}

Gene expression data and corresponding clinical data of LUAD patients were downloaded from the Gene Expression Omnibus (GEO) database. The GSE31210 dataset was used to verify whether PLOD $1 / 2 / 3$ were independent prognostic indicators. In GSE31210 dataset, some patients were excluded from the prognosis analysis due to incomplete clinical data, incomplete resection, or adjuvant therapy. A total of 204 LUAD patients from the GSE31210 dataset were enrolled.

\section{Statistical analysis}

Overall survival (OS) was calculated from the date of randomization until the date of death from any cause or last follow-up. Progression-free survival (PFS) was calculated from the date of randomization until the date of progression or death from any cause or last follow-up. Post-progression survival (PPS) was defined as the time from the date of first progression to second progression or deaths. Relapsefree survival (RFS) was defined as the time from surgery to disease relapse or last follow-up.

According to clinical samples, we evaluated the differential expression of PLODs between NSCLC tissues and adjacent normal tissues with paired $t$-test. The Kaplan-Meier method was used to carry out survival analyses, and the log-rank test was calculated to evaluate the differences in survival. On the basis of clinical data of the GSE31210 dataset, the Cox proportional hazards regression model was applied to perform univariate and multivariate analyses. SPSS 25.0 software (SPSS, Chicago, IL) and GraphPad Prism 8.0 were applied to perform the analyses mentioned above. The reported $\mathrm{P}$ values were two-sided, and P-value $<0.05$ was considered statistically significant.

\section{Results}

\section{Transcriptional levels of PLOD family members in patients with NSCLC}

In accordance with the Oncomine database, we investigated the mRNA expression levels of PLODs in different cancers (e.g., bladder, liver, and lung cancers; Figure 1A). We also further analyzed the transcriptional levels of PLODs between tumor and normal tissues in different subtypes of NSCLC, including LUAD, LUSC, and large cell lung carcinoma (Table 1). In Garber's dataset (24), the expression levels of PLOD1 in LUAD (fold change: 1.998), LUSC (fold change: 1.562), and large cell lung carcinoma (fold change: 4.286) were significantly up-regulated compared with those in normal tissues. Similar to the results of Garber's dataset, Hou et al. (25) found that PLOD1 is significantly up-regulated in LUAD (fold change: 1.493) and LUSC (fold change: 1.604). Regarding the mRNA expression change of PLOD2, Garber et al. (24), Landi et al. (26), and Talbot et al. (27) observed that PLOD2 is significantly overexpressed in large cell lung carcinoma (fold change: 4.031), LUAD (fold change: 3.338), and LUSC (fold change: 3.470). Moreover, Garber et al. (24), Landi et al. (26) and Talbot et al. (27) observed that PLOD3 is significantly overexpressed in different subtypes of NSCLC (large cell lung 
A

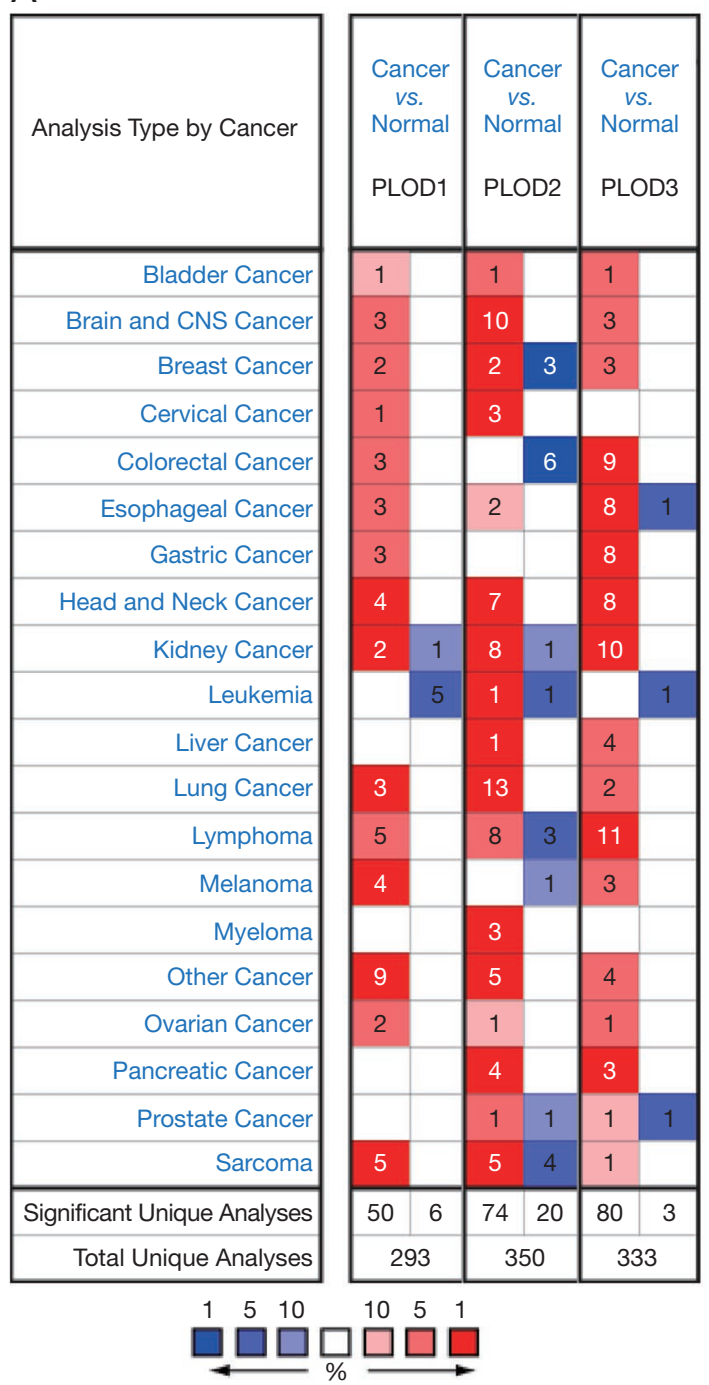

B
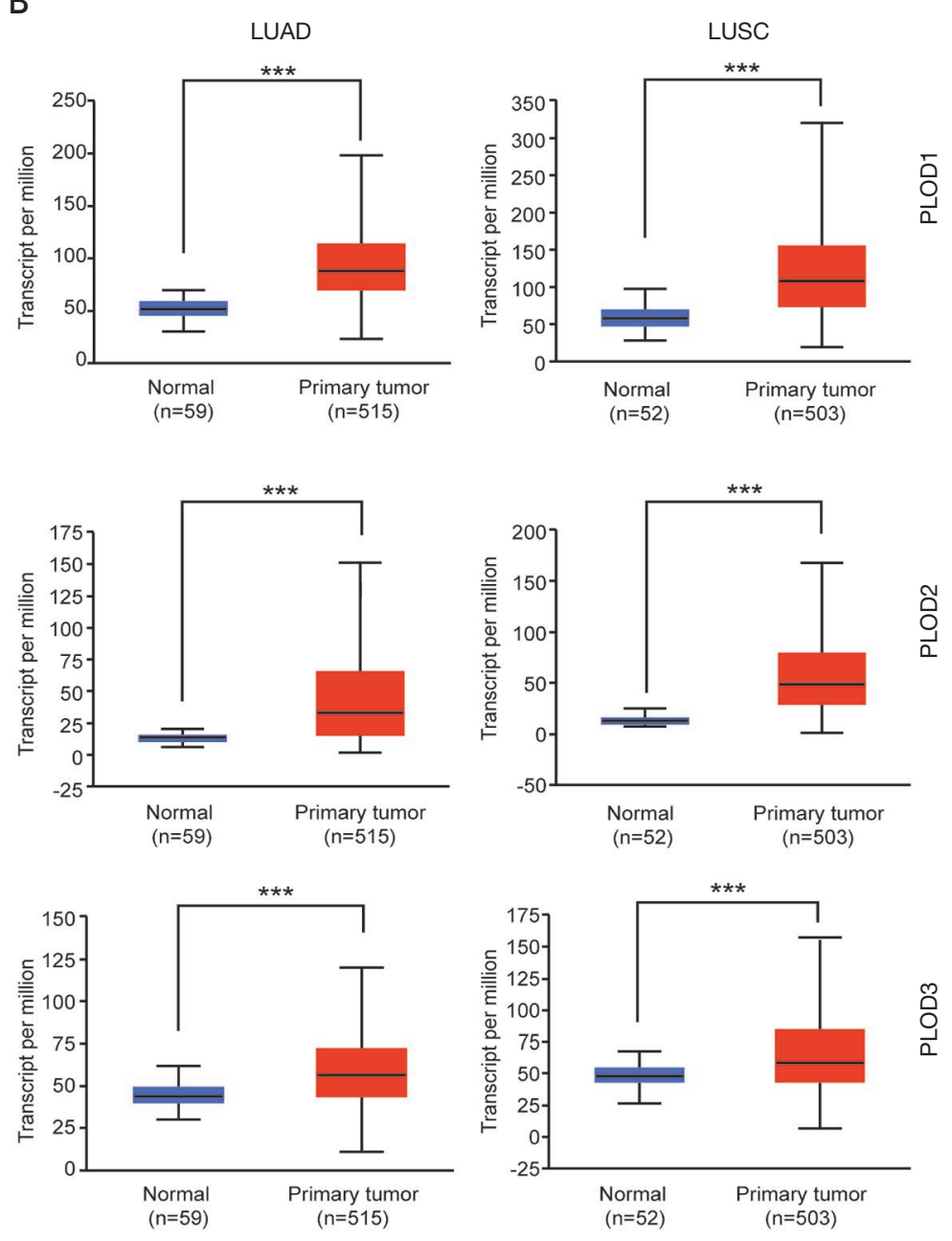

Figure 1 Transcriptional levels of PLOD family members in lung cancer. (A) Expression levels of PLOD1, PLOD2, and PLOD3 in different types of cancers (ONCOMINE database). The parameters were set as follows: P-value: 0.01; fold change: 1.5; gene rank: 10\%; and data type: mRNA. (B) mRNA expression levels of PLODs in normal and cancer tissues in LUAD and LUSC. ${ }^{* * *}, \mathrm{P}<0.001$. PLOD, procollagen-lysine,2-oxoglutarate 5-dioxygenase; LUAD, lung adenocarcinoma; LUSC, lung squamous cell carcinoma.

carcinoma: fold change, 2.471; LUAD: fold change, 1.111; LUSC: fold change, 1.508).

Using the UALCAN website, the mRNA expression levels of PLODs in normal and cancer tissues and different tumor stages were explored. Compared with those in normal tissues, the expression levels of PLOD $1 / 2 / 3$ in LUAD and LUSC were significantly higher (Figure 1B), and these results were consistent with those observed in the Oncomine database. The expression levels of PLODs in different tumor stages were also investigated in LUAD and LUSC, which indicated that the expression levels of PLOD1/2/3 in cancer tissues at different tumor stages were significantly higher than those in normal tissues (Figure 2). However, the expression levels of PLOD3 in stage IV LUSC and normal tissues were not significantly different, which may be attributed to the small sample size of the stage IV tumor (Figure $2 F$ ). We found that the mRNA expression levels of PLOD1/2/3 among different tumor stages were not significant.

In addition, according to the Human Protein Atlas 
Table 1 The significant changes of PLODs expression in mRNA level between NSCLC and normal lung tissues (ONCOMINE)

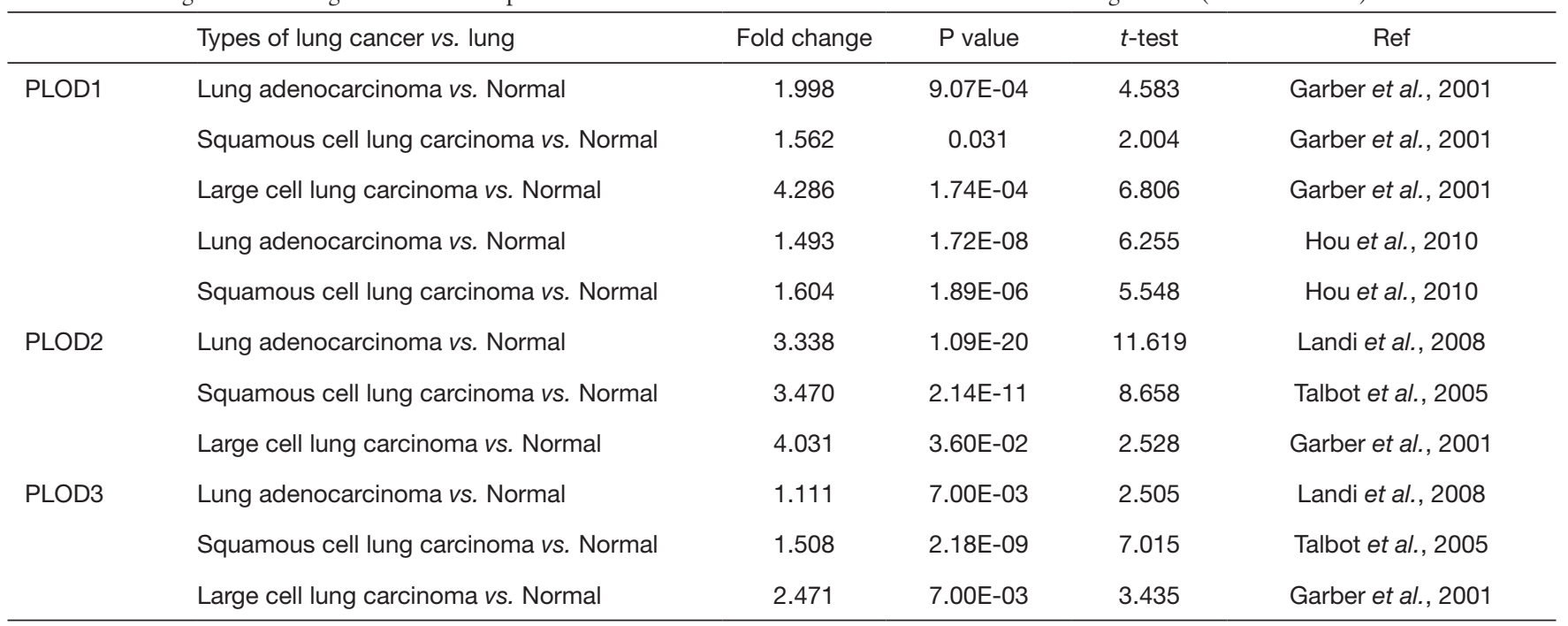

PLOD, procollagen-lysine, 2-oxoglutarate 5-dioxygenase; NSCLC, non-small-cell lung cancer.

A

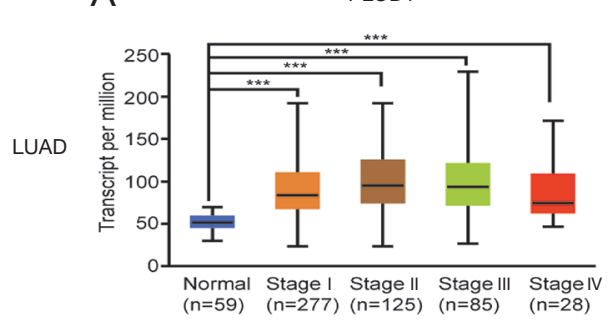

D

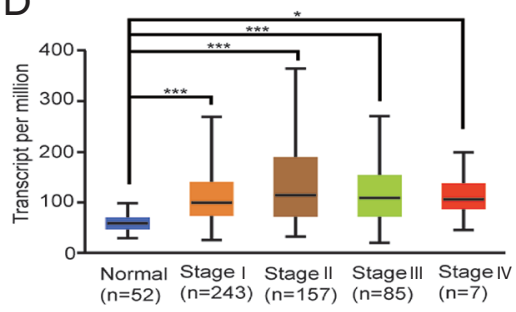

B

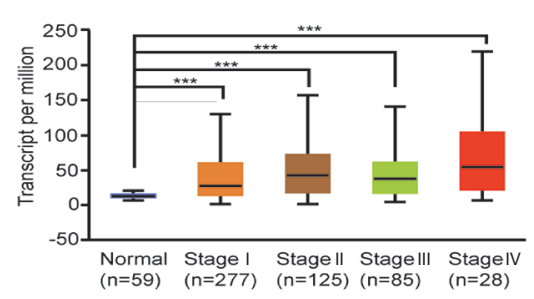

E

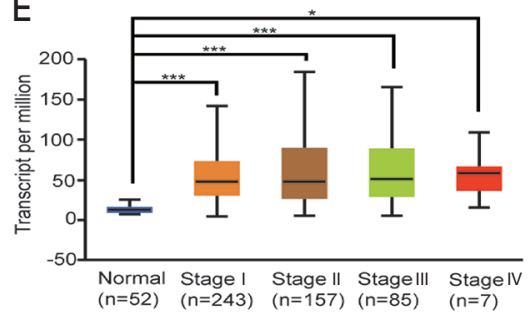

C

PLOD3

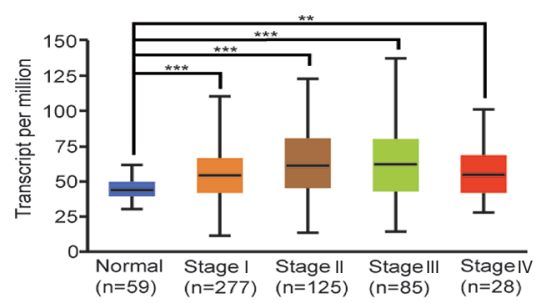

$\mathrm{F}$

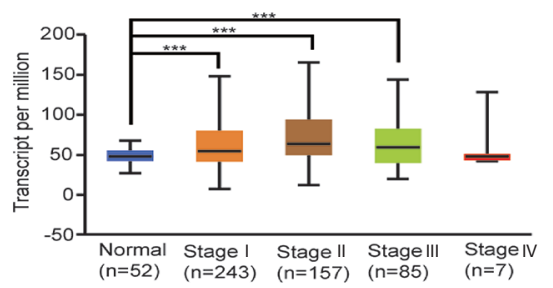

Figure 2 Correlation between the mRNA expression of PLODs and tumor stage in patients with lung cancer (UALCAN). Correlation between PLOD expression and tumor stage in (A,B,C) LUAD and (D,E,F) LUSC. * $\mathrm{P}<0.05$; **, $\mathrm{P}<0.01$; *** $\mathrm{P}<0.001$. PLOD, procollagenlysine,2-oxoglutarate 5-dioxygenase; LUAD, lung adenocarcinoma; LUSC, lung squamous cell carcinoma.

(https://www.proteinatlas.org) (19), we further analyzed the protein expression patterns of PLOD1/2/3 in LUAD and LUSC. As shown in Figure 3, PLOD1/2/3 proteins were not detected in normal tissues, whereas low and moderate expression levels of PLOD1/2/3 proteins were detected in LUAD and LUSC. Our results showed that the mRNA and protein expression levels of PLOD1/2/3 were up-regulated in patients with NSCLC.

\section{Correlation of the transcriptional levels of PLOD family} members with the TP53 mutation in patients with NSCLC

In accordance with the UALCAN website, we analyzed the correlations of the mRNA levels of PLOD1/2/3 with TP53 mutation in patients with LUAD and LUSC. The results showed that regardless of whether TP53 was mutated, the expression levels of PLOD1/2/3 were significantly high in 
PLOD1

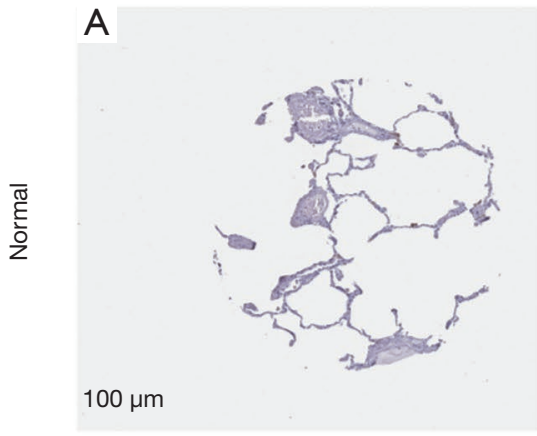

Staining: not detect Antibody: HPA049137

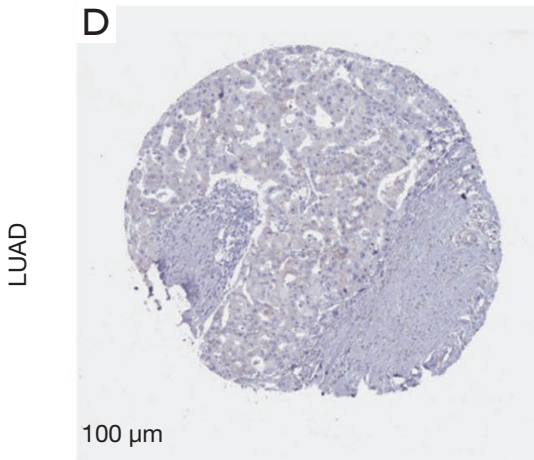

Staining: low Antibody: HPA049137

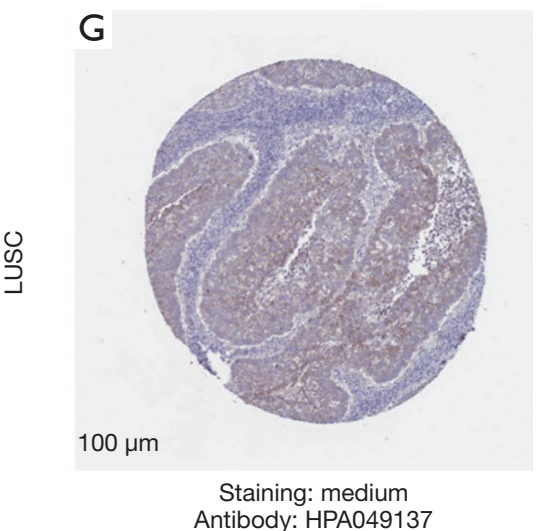

PLOD2

B

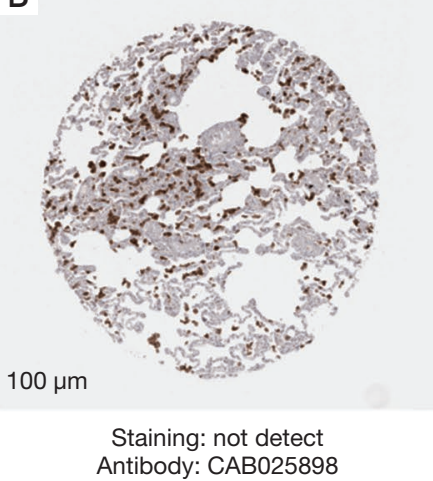

$E$

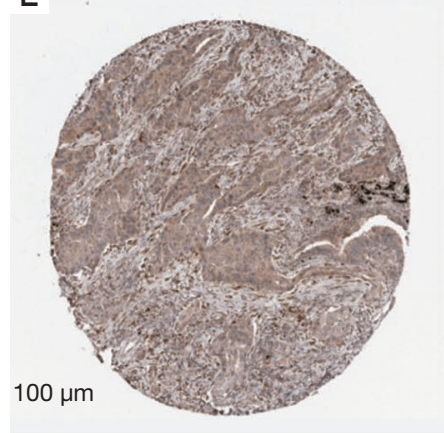

Staining: medium Antibody: CAB025898

$\mathrm{H}$

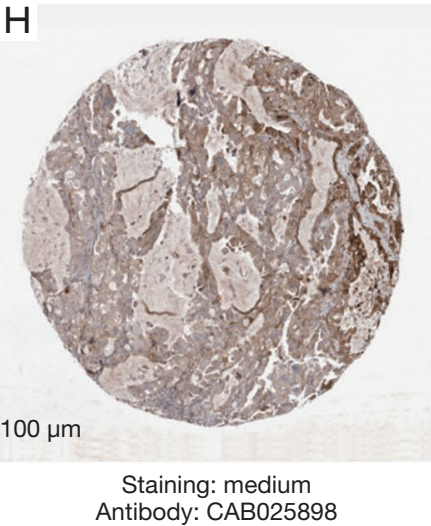

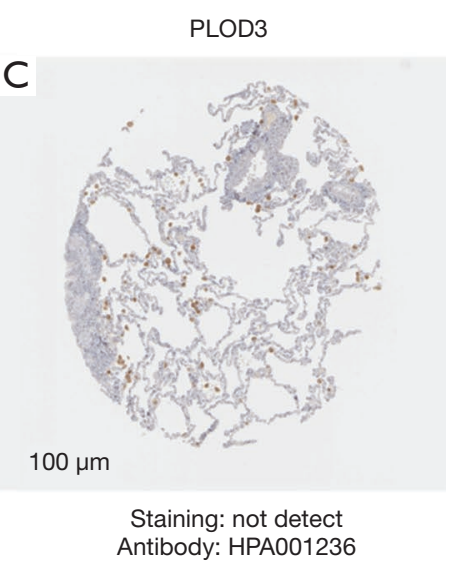

$\mathrm{F}$

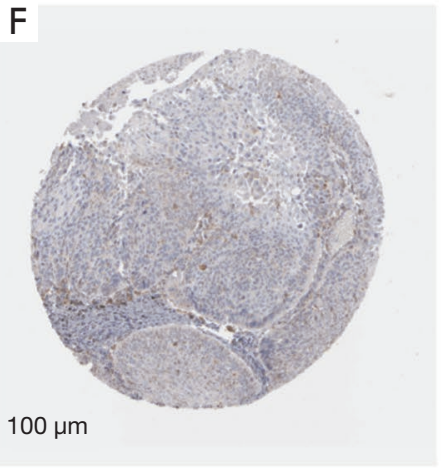

Staining: medium Antibody: HPA001236

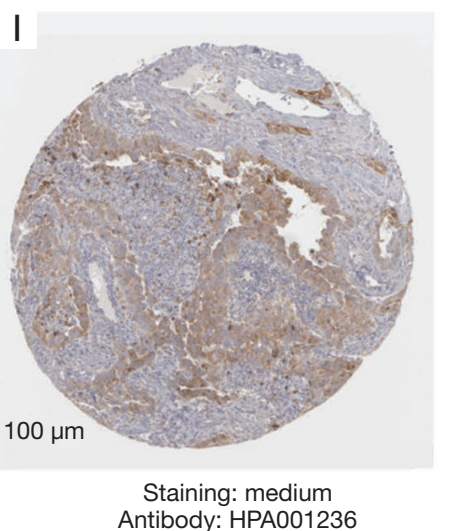

Figure 3 Representative immunohistochemistry images of PLOD1, PLOD2, and PLOD3 in tumors and normal lung tissues (Image credit: Human Protein Atlas, https://www.proteinatlas.org). (A,B,C) PLOD1/2/3 proteins are not expressed in normal tissues. (D,E,F). Moderate protein expression levels of PLOD2/3 were detected in LUAD tissues, whereas low protein expression levels of PLOD1 were found in LUAD tissue. (G,H,I) Moderate protein expression levels of PLOD1/2/3 were observed in LUSC tissues. PLOD, procollagen-lysine, 2-oxoglutarate 5-dioxygenase; LUAD, lung adenocarcinoma; LUSC, lung squamous cell carcinoma. 
LUAD (Figure S1A,B,C) and LUSC (Figure S1D,E,F). We also found that the expression levels of PLOD1/2/3 from tumor tissues with the TP53 mutation were significantly higher than those without the TP53 mutation in LUAD (Figure S1A,B,C), whereas the TP53 mutation had no significant effect on the mRNA expression levels of PLOD1/2/3 in LUSC (Figure S1D,E,F).

\section{Prognostic values of PLOD family members in patients with NSCLC}

Using the Kaplan-Meier Plotter tool, we evaluated the prognostic values of the mRNA expression levels of PLOD $1 / 2 / 3$ in patients with lung cancer. The results indicated that the increased mRNA expression levels of PLOD $1 / 2 / 3$ were significantly related to inferior OS and PFS in patients with LUAD (Figure $4 A, B, C, D, E, F$ ). The high expression levels of PLOD1/2/3 were not significantly related to PPS in patients with LUAD (Figure 4G,H,I). For patients with LUSC, the correlations between the mRNA expression levels of PLOD1/2/3 and survival (OS, PFS, and PPS) were not statistically significant (Figure 5).

To further verify whether the PLOD $1 / 2 / 3$ are independent prognostic factors in patients with LUAD, we analyzed LUAD patients from the GSE31210 dataset and evaluated the prognostic impact of PLOD family members by establishing a Cox proportional hazards model. The univariate analysis showed that TNM stage and PLOD1/2/3 expression were significantly associated with OS and RFS (Tables 2,3). Further multivariate analysis confirmed that PLOD 1 and PLOD3 expression were independent risk factors for OS and RFS in LUAD patients (Tables 2,3). We did not observe that PLOD2 was an independent risk factor for OS and RFS, whereas the P value was nearly 0.05 for OS, which need to be further verified by a large scale of clinical samples.

\section{Validation of PLODs expression in NSCLC tissues}

To further validate the expression levels of PLOD1/2/3, RT-qPCR was performed to examine the mRNA levels of PLOD1/2/3 in 33 NSCLC tissues and matched adjacent normal tissues collected in our hospital. In addition to PLOD2 in LUAD, the expression levels of PLOD1/2/3 in NSCLC tissues were significantly higher than those in matched normal tissues (Figure 6A,B,C). Similar to the results of the bioinformatic analysis, the expression levels of PLODs were not significantly related to survival in patients with LUSC (Figure 6D,E,F). However, unlike the results of the bioinformatic analysis, statistically significant differences were not observed between the mRNA expression levels of PLODs and OS in patients with LUAD (Figure 6D,E,F).

\section{Genetic alterations in PLOD family members and their correlations with OS and DFS in patients with NSCLC}

We investigated the genetic alterations in PLOD1/2/3 and their correlations with OS and DFS in patients with LUAD and LUSC by using the cBioPortal tool. The results showed that PLODs were altered in 99 out of 510 patients with LUAD, for a mutation rate of $19.41 \%$ (Figure $7 A$ ). The frequency of PLOD3 gene alteration was highest among PLOD family members (PLOD1: 6\%, PLOD2: 8\%, PLOD3: 12\%; Figure 7B). For patients with LUSC, the results revealed that PLODs were altered in 181 out of 484 patients, for a mutation rate of $37.40 \%$ (Figure S2A). In contrast, PLOD2 ranked highest among the three PLOD genes (PLOD1: 5\%; PLOD2: 25\%; PLOD3: 14\%) in terms of the frequency of genetic alteration in LUSC (Figure S2B). Subsequently, the correlations among PLOD1, PLOD2 and PLOD3 were evaluated in LUAD and LUSC by analyzing the mRNA expression levels of PLODs. Pearson's correlation coefficient was calculated. In LUAD, PLOD1 was significantly and positively correlated with PLOD2 and PLOD3 and PLOD2 was significantly and positively correlated with PLOD3 (Figure 7C). In LUSC, PLOD1 was significantly and positively correlated with PLOD2 and PLOD3 and PLOD2 was significantly and positively correlated with PLOD3 (Figure S2C), which was similar to the results from LUAD. The Kaplan-Meier plot and the log-rank test indicated that the correlation between the genetic alterations of PLODs and survival was not statistically significant in patients with LUAD (Figure $7 D$, OS: $\mathrm{P}=0.164$; Figure $7 E$, DFS: $\mathrm{P}=0.849$ ). For patients with LUSC, the genetic alterations of PLODs had significant relevance to favorable OS (Figure S2D, $\mathrm{P}=0.035$ ) and no significant relevance to DFS (Figure S2E, $\mathrm{P}=0.495$ ).

\section{Functional enrichment analysis of co-expressed genes of PLODs in NSCLC}

We obtained the co-expressed genes of PLODs from cBioPortal. The co-expressed genes with Spearman correlation coefficients $>0.4$ were selected to conduct GO and KEGG pathway analyses in Metascape. As shown in 

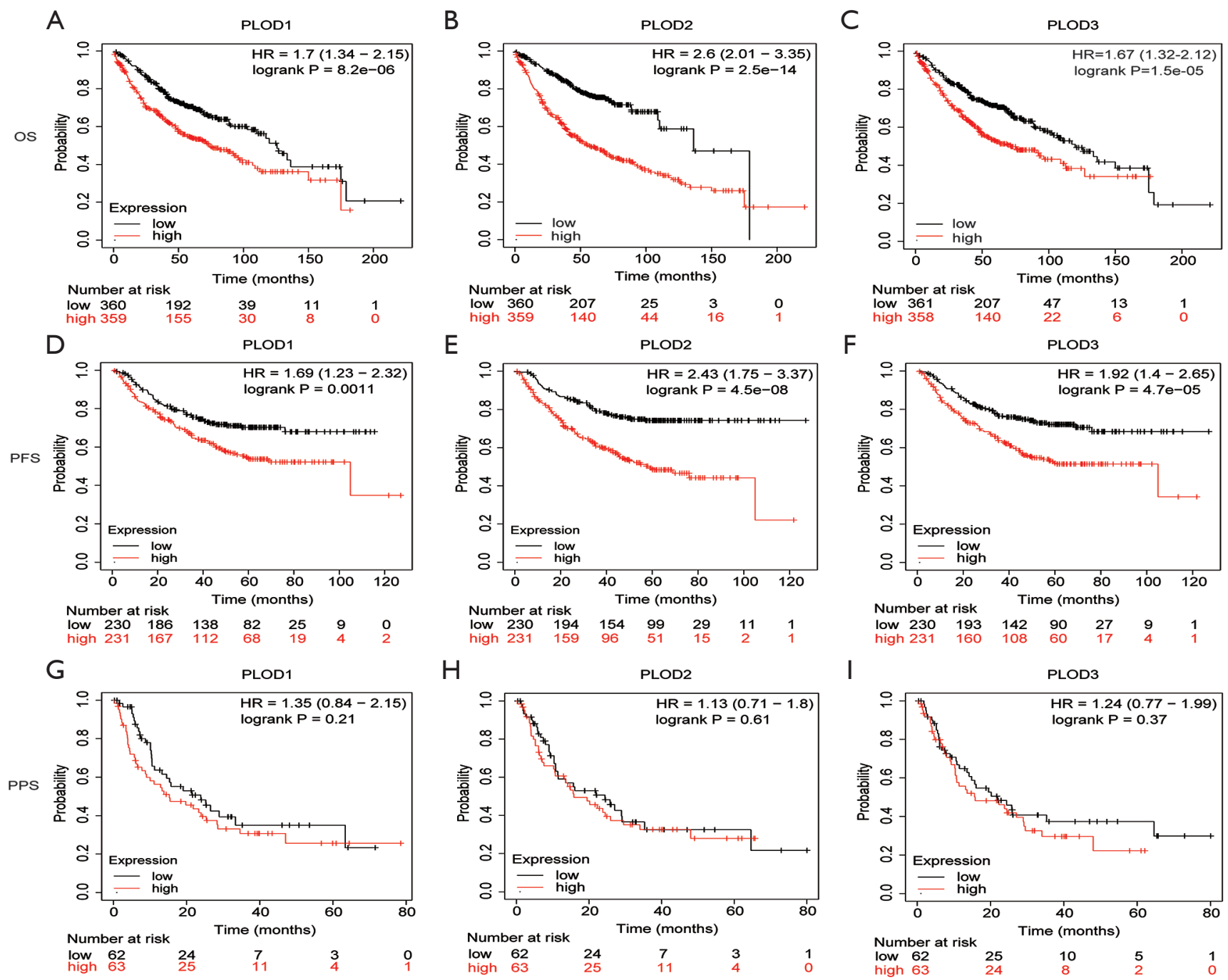

Figure 4 Prognostic value of the mRNA expression of PLODs in LUAD (Kaplan-Meier plotter). Relationships between the mRNA expression of PLODs and (A,B,C) OS, (D,E,F) PFS, and (G,H,I) PPS. PLOD, procollagen-lysine,2-oxoglutarate 5-dioxygenase; LUAD, lung adenocarcinoma; OS, overall survival; PFS, progression-free survival; PPS, post-progression survival; HR, hazard ratio.

Figure 8, biological processes, including cell division, DNAdependent DNA replication, microtubule-based process, DNA repair, meiotic cell cycle process, DNA conformation change, positive regulation of cell cycle process, collagen fibril organization, metaphase plate congression, attachment of spindle microtubules to kinetochore, DNA integrity checkpoint, supramolecular fiber organization, response to hypoxia, cytokinesis, and regulation of DNA metabolic process, were prominently regulated by the co-expression genes of PLODs in LUAD (Figure 8A). Cellular components, which included the chromosome centromeric region, spindle, microtubule-organizing center, condensed nuclear chromosome, and spindle midzone, had evident significant correlations with the co-expression genes of PLODs in LUAD (Figure 8B). These co-expressed genes also had a primary influence on molecular functions, such as catalytic activity acting on DNA, microtubule binding, histone kinase activity, cell adhesion molecule binding, damaged DNA binding, DNA replication origin binding, ECM structural constituent, kinase binding, anaphase-promoting complex binding, L-ascorbic acid binding, and chromatin binding (Figure 8C). Subsequently, we further performed a KEGG pathway analysis and found that the functions of these co-expressed genes were involved in multiple pathways, 

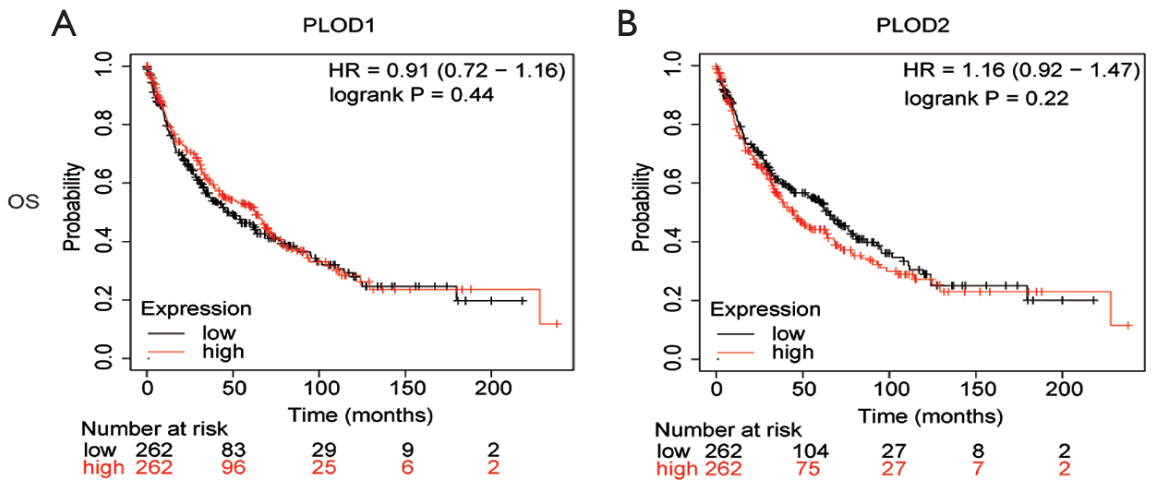

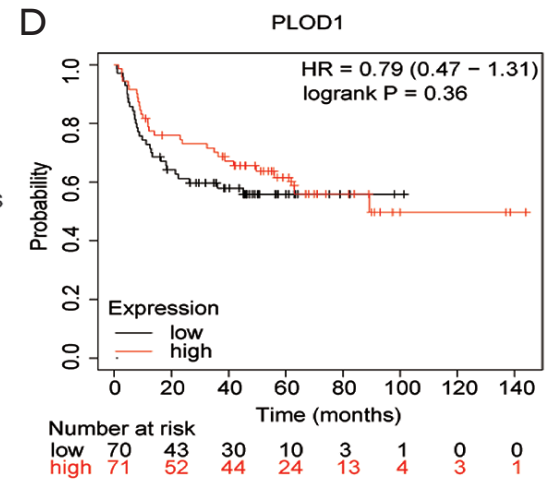

$\mathrm{E}$

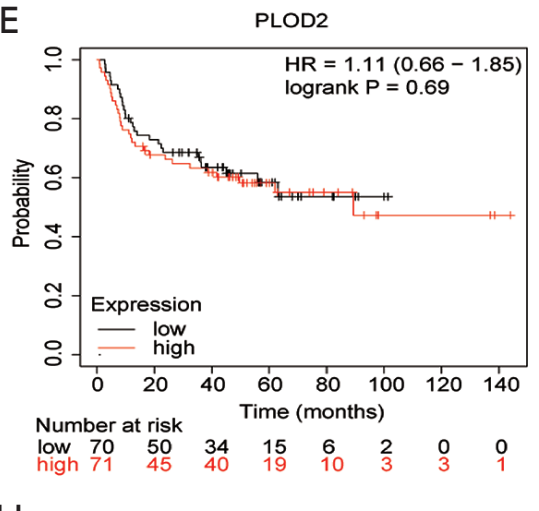

G

PLOD1

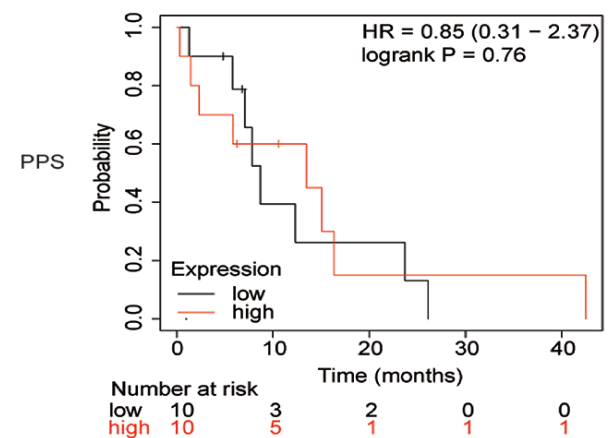

$\mathrm{H}$

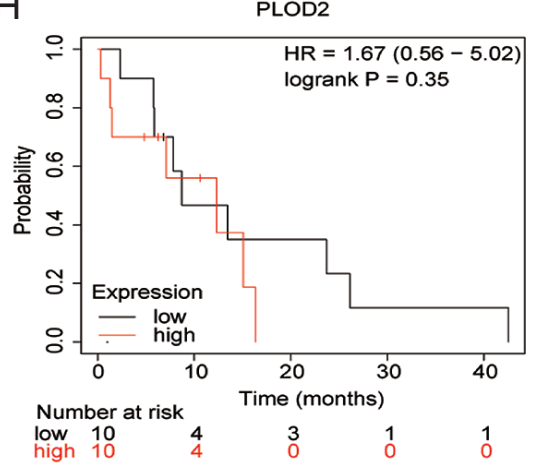

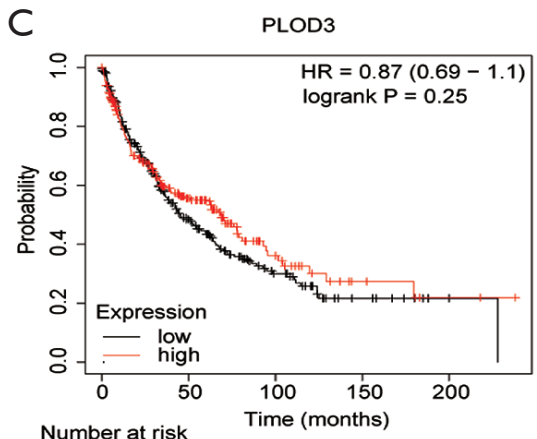

C

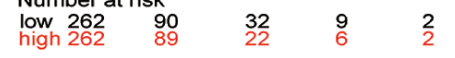

$\mathrm{F}$

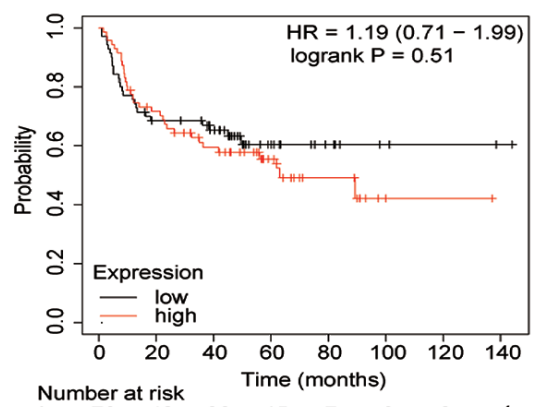

I

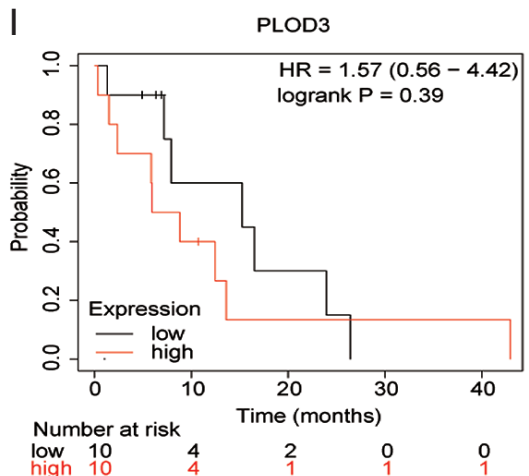

Figure 5 Prognostic value of the mRNA expression of PLODs in LUSC (in Kaplan-Meier plotter). Relationships between the mRNA expression of PLODs and (A,B,C) OS, (D,E,F) PFS, and (G,H,I) PPS. PLOD, procollagen-lysine, 2-oxoglutarate 5-dioxygenase; LUAD, lung adenocarcinoma; OS, overall survival; PFS, progression-free survival; PPS, post-progression survival; HR, hazard ratio.

including the cell cycle, DNA replication, glycolysis/ gluconeogenesis, homologous recombination, central carbon metabolism in cancer, p53 signaling pathway, pathways in cancer, and PI3K-Akt signaling pathway (Figure $8 D$ ). In addition, we also performed enrichment analyses of the co-expressed genes of PLODs in LUSC (Figure S3). In terms of biological processes, the co-expressed genes of PLODs from LUSC were enriched in extracellular structure organization, protein hydroxylation, cell-substrate adhesion, posttranslational protein modification, respiratory system development, response to hypoxia, basement membrane assembly, protein folding, collagen metabolic process, peptidyl-lysine oxidation, and endoderm development (Figure S3A). The cellular components of these genes from LUSC were observed in the endoplasmic reticulum lumen, ECM, focal adhesion, Golgi cisterna membrane, and endoplasmic reticulum chaperone complex (Figure S3B). The molecular functions of these genes from LUSC were 
Table 2 Univariate and multivariate analyses of overall survival for patients with lung adenocarcinoma

\begin{tabular}{|c|c|c|c|c|c|c|}
\hline Variable & \multicolumn{3}{|c|}{ Univariate analysis } & \multicolumn{3}{|c|}{ Multivariate analysis } \\
\hline Gender (Male vs. female) & 1.686 & $0.818-3.476$ & 0.157 & - & - & - \\
\hline Age, years (>60 vs. $\leq 60)$ & 1.271 & $0.617-2.619$ & 0.515 & - & - & - \\
\hline Smoking (Smoker vs. nonsmoker) & 1.908 & $0.918-3.966$ & 0.084 & - & - & - \\
\hline TNM stage (II vs. I) & 4.297 & $2.092-8.828$ & $<0.001$ & 3.290 & $1.568-6.900$ & 0.002 \\
\hline PLOD1 expression (High vs. low) & 4.312 & $1.762-10.555$ & 0.001 & 3.290 & $1.312-8.247$ & 0.011 \\
\hline \multicolumn{7}{|l|}{ PLOD2 Group } \\
\hline TNM stage (II vs. I) & 4.297 & $2.092-8.828$ & $<0.001$ & 3.377 & $1.593-7.158$ & 0.001 \\
\hline TNM stage (II vs. I) & 4.297 & $2.092-8.828$ & $<0.001$ & 3.664 & $1.763-7.614$ & 0.001 \\
\hline PLOD3 expression (High vs. low) & 2.931 & $1.304-6.589$ & 0.009 & 2.376 & $1.043-5.413$ & 0.039 \\
\hline
\end{tabular}

HR, hazard ratio; Cl, confidence interval; PLOD1, Procollagen-lysine, 2-oxoglutarate 5-dioxygenase 1; PLOD2, Procollagen-lysine, 2-oxoglutarate 5-dioxygenase 2; PLOD3, Procollagen-lysine, 2-oxoglutarate 5-dioxygenase 3.

Table 3 Univariate and multivariate analyses of relapse-free survival for patients with lung adenocarcinoma

\begin{tabular}{|c|c|c|c|c|c|c|}
\hline Variable & \multicolumn{3}{|c|}{ Univariate analysis } & \multicolumn{3}{|c|}{ Multivariate analysis } \\
\hline Age, years (>60 vs. $\leq 60)$ & 1.570 & $0.908-2.714$ & 0.106 & - & - & - \\
\hline Smoking (Smoker vs. nonsmoker) & 1.430 & $0.837-2.443$ & 0.190 & - & - & - \\
\hline TNM stage (II vs. I) & 3.443 & $2.977-5.996$ & $<0.001$ & 3.017 & $1.687-5.397$ & $<0.001$ \\
\hline PLOD1 expression (High vs. low) & 2.391 & $1.342-4.259$ & 0.003 & 1.860 & $1.019-3.395$ & 0.043 \\
\hline \multicolumn{7}{|l|}{ PLOD2 Group } \\
\hline \multicolumn{7}{|l|}{ PLOD3 Group } \\
\hline TNM stage (II vs. I) & 3.443 & $2.977-5.996$ & $<0.001$ & 2.818 & $1.607-4.941$ & $<0.001$ \\
\hline PLOD3 expression (High vs. low) & 3.433 & $1.867-6.313$ & $<0.001$ & 2.961 & $1.597-5.490$ & 0.001 \\
\hline
\end{tabular}

HR, hazard ratio; CI, confidence interval; PLOD1, Procollagen-lysine, 2-oxoglutarate 5-dioxygenase 1; PLOD2, Procollagen-lysine, 2-oxoglutarate 5-dioxygenase 2; PLOD3, Procollagen-lysine, 2-oxoglutarate 5-dioxygenase 3. 

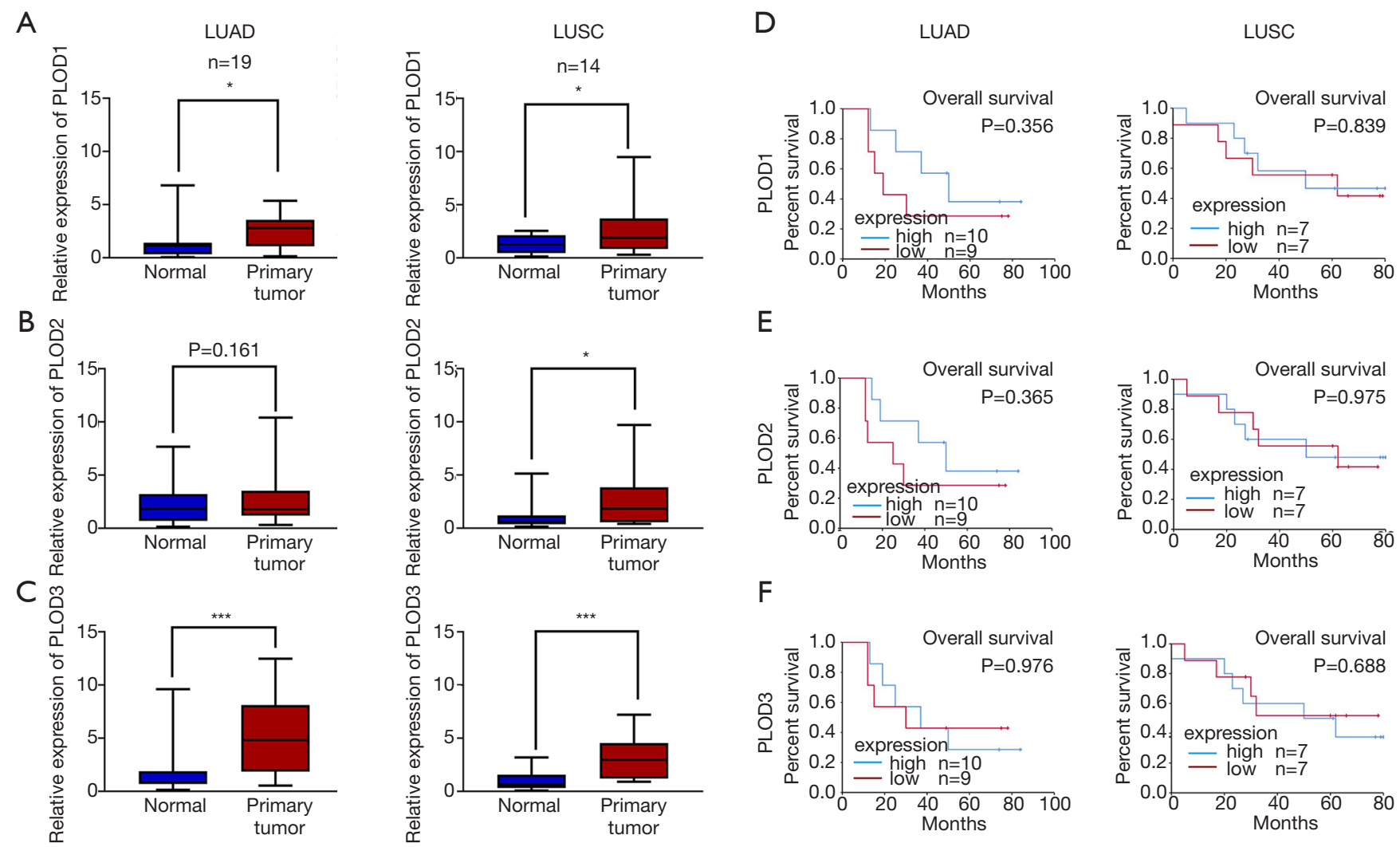

E
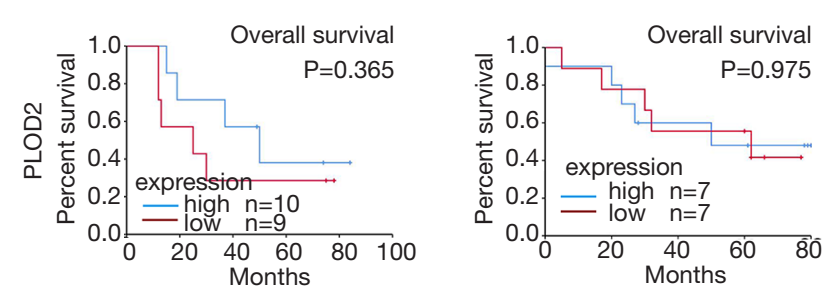

F
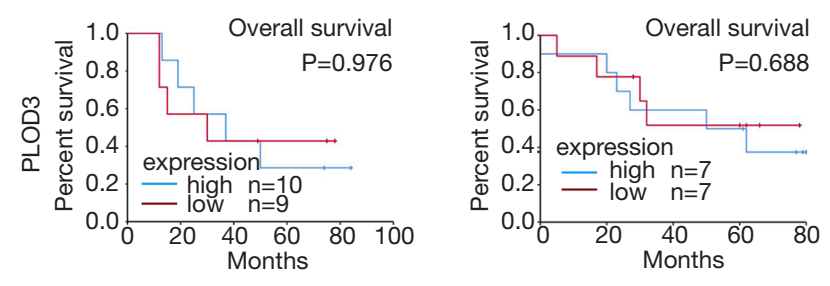

Figure 6 Expression and prognostic value of PLODs in clinical samples, including LUAD and LUSC. (A,B,C) mRNA expression of PLOD1/2/3 in LUAD and LUSC tissues and matched normal tissues analyzed by RT-qPCR. (D,E,F) Relationship between the mRNA expression of PLODs and OS in LUAD and LUSC. *, $\mathrm{P}<0.05$; ${ }^{* *}, \mathrm{P}<0.001$. PLOD, procollagen-lysine,2-oxoglutarate 5-dioxygenase; LUAD, lung adenocarcinoma; LUSC, lung squamous cell carcinoma; OS, overall survival.

involved in L-ascorbic acid binding, ECM structural constituent, disulfide oxidoreductase activity, calcium ion binding, transferase activity, transferring hexosyl groups, protein-lysine 6-oxidase activity, cell adhesion molecule binding, and collagen binding (Figure S3C). For the KEGG analysis of these genes from LUSC, we found three related pathways: ECM-receptor interaction, protein processing in endoplasmic reticulum, and glycosaminoglycan biosynthesischondroitin sulfate/dermatan sulfate (Figure S3D).

\section{Correlation analysis between PLOD expression and tumor immunity}

Using the TIMER database, we analyzed the relationship between the transcriptional expression of PLODs and various immune-infiltrating cells in LUAD and LUSC. The results showed that the expression levels of PLOD1/2/3 had significant relevance to the infiltrating levels of some immune-related cells (Figure S4), whereas the correlations between PLOD expression and immuneinfiltrating cells were not evident. In addition, we further analyzed whether PLOD expression was associated with some immune-related molecules, including $\mathrm{PD}$ L1 (CD274), CXCL10, GZMA, and GZMB, in LUAD and LUSC (Figure S5). In LUAD, the results showed that CD274 was significantly and positively correlated with PLOD1, PLOD2, and PLOD3; CXCL10 was significantly and positively correlated with PLOD1, PLOD2, and PLOD3; GZMA was significantly and positively correlated with PLOD2; and GZMB was significantly and positively correlated with PLOD1, PLOD2, and PLOD3 (Figure S5A,C,E,G). In LUSC, PLOD1 was significantly and negatively correlated with GZMA and GZMB (Figure S5F,H), and PLOD2 was significantly and positively correlated with CXCL10 (Figure S5D). Notably, the correlations between PLOD2 


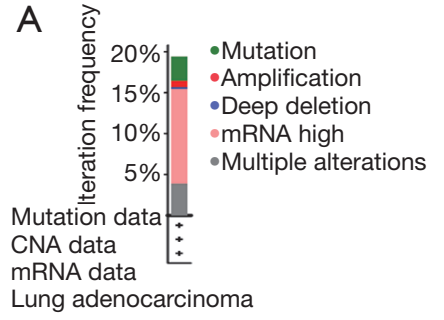

C

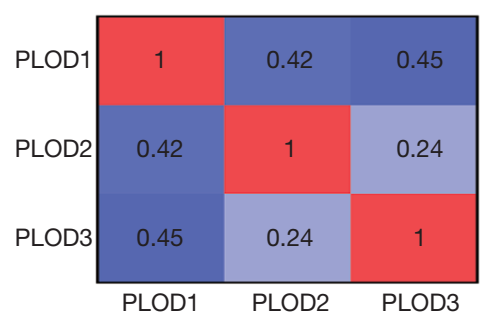

B

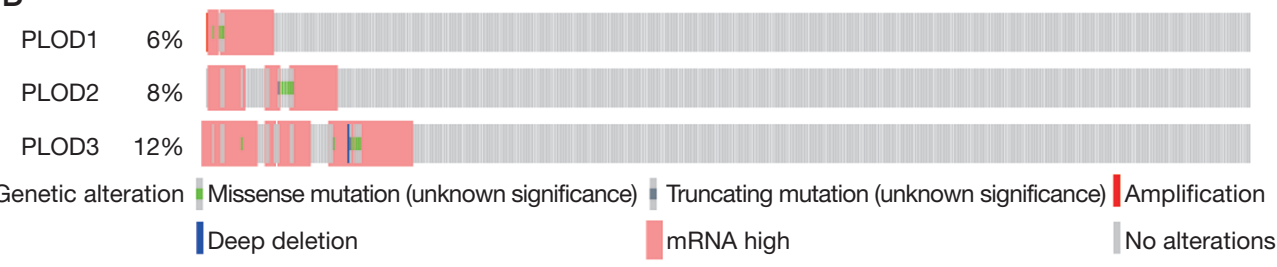

D

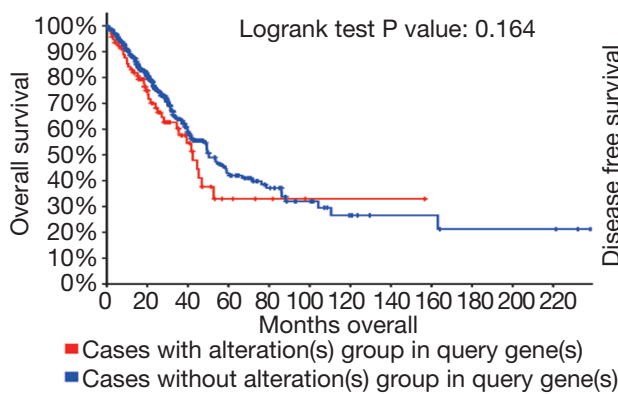

E

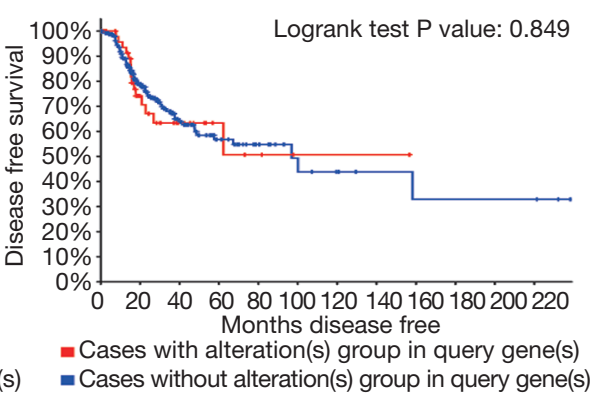

Figure 7 Expression mutation analysis of PLODs in LUAD (cBioPortal). (A) Summary of genetic alterations in PLODs. (B) Genetic alterations in individual PLOD family members. (C) Pearson correlation among PLOD1, PLOD2, and PLOD3. (D,E) Correlation between genetic alterations and survival (OS and DFS). PLOD, procollagen-lysine,2-oxoglutarate 5-dioxygenase; LUSC, lung squamous cell carcinoma; OS, overall survival; DFS, disease-free survival.

expression and immune-related genes were more obvious compared with those between PLOD1/3 expression and immune-related genes in LUAD.

\section{Discussion}

The importance of PLOD family genes has drawn increasing attention due to their critical role in the synthesis of collagen. Previous studies indicated that PLODs are involved in various malignant behaviors of tumors, such as proliferation, invasion, and metastasis $(16,17,28,29)$. The important roles of PLODs have been reported in the development and progression of several tumors, whereas the prognostic values and potential functions of PLODs have yet to be comprehensively elucidated in lung cancer. We performed bioinformatic analyses, including transcriptional expression, genetic alteration, prognostic value, potential function, and immunity-related evaluations, for PLOD family members in NSCLC. In this study, we found that the mRNA and protein expression levels of PLOD1/2/3 were up-regulated in NSCLC tissues. Furthermore, RT-qPCR was used to validate the mRNA expression differences of PLODs in NSCLC tissues and matched normal lung tissues. Based on bioinformatic analysis, we observed that a high PLOD1/2/3 expression level has significant relevance for poor OS and PFS in patients with LUAD. In addition, PLOD1 and PLOD3 could serve as independent risk factors for survival in LUAD patients. Interestingly, the clinical samples collected in our hospital revealed that the prognostic values of PLOD1/2/3 were not statistically significant in patients with LUAD. We considered that the results of the lack of survival difference in the clinical samples are attributed to the small sample size.

In recent years, the important roles of PLOD family members in tumor biological behavior and prognosis have received increasing attention. At present, the correlation between PLOD1 and lung cancer has not been investigated. In other types of tumors, the abnormal expression of PLOD1 is linked with cancer progression and prognosis $(14,30)$. Yamada et al. (30) found that PLOD1 played an essential role in the proliferation, migration, and invasion of bladder cancer and might be a target of treatment for bladder cancer. Yamada and colleagues have also demonstrated that high PLOD1 expression was significantly associated with inferior survival. Our study also found that high expression of PLOD1 was significantly related to poor OS and PFS in patients with LUAD. As the key enzyme catalyzing the hydroxylation of lysyl to form hydroxylysine, PLOD2 critically affects the formation of stable collagen 
A

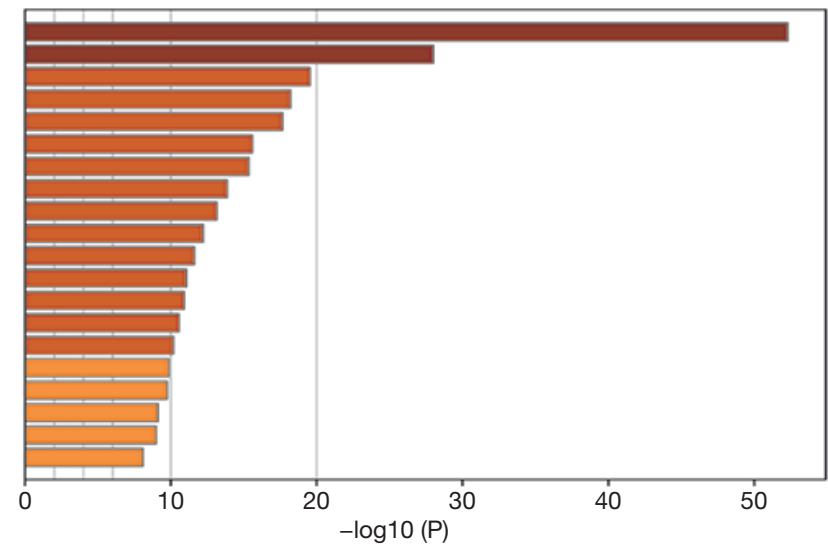

B

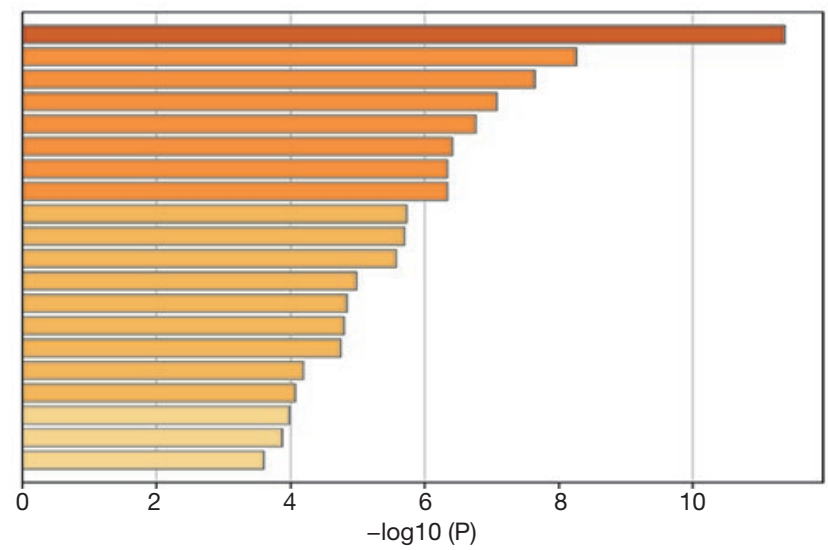

C

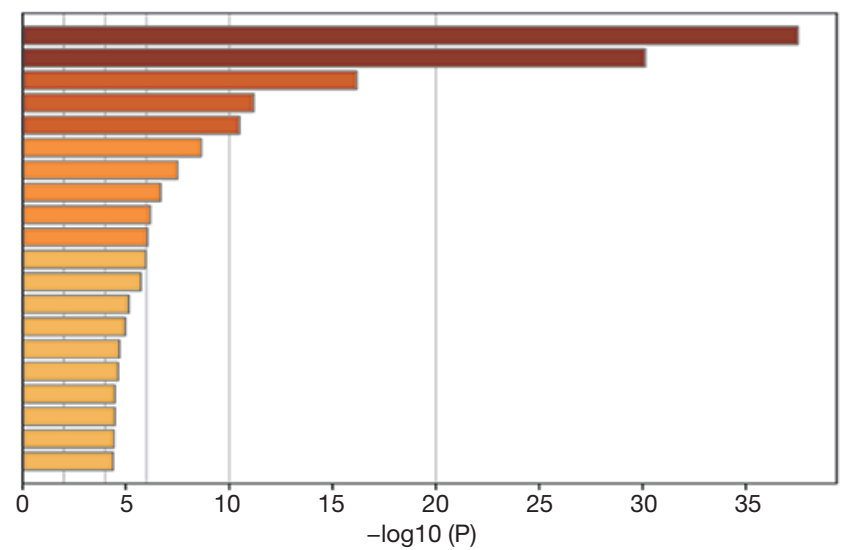

$-\log 10(P)$
GO: 0051301: cell division

GO: 0006261: DNA-dependent DNA replication

GO: 0007017: microtubule-based process GO: 0006281: DNA repair

GO: 1903046: meiotic cell cycle process

GO: 0071103: DNA conformation change

GO: 0090068: positive regulation of cell cycle process

GO: 0030199: collagen fibril organization

GO: 0051310: metaphase plate congression

GO: 0051310: metaphase plate congression

GO: 0008608: attachment of spindle mic

GO: 0031570: DNA integrity checkpoint

GO: 0097435: supramolecular fiber org

GO: 0000910: cytokinesis

GO: 0051052: regulation of DNA metabolic process

GO: 0034502: protein localization to chromosome

GO: 0000727: double-strand break repair via break-induced replication

GO: 0031145: anaphase-promoting complex-dependent catabolic process

G: 0009124: nucleoside monophosphate biosynthetic process

GO: 0006271: DNA strand elongation involved in DNA replication

GO: 0140097: catalytic activity, acting on DNA

GO: 0008017: microtubule binding

GO: 0035173: histone kinase activity

GO: 0003684: damaged DNA binding

GO: 0003688: DNA replication origin binding

GO: 0005201: extracellular matrix structural constituent

GO: 0019900: kinase binding

GO: 0010997: anaphase-promoting complex binding

GO: 0031418: L-ascorbic acid binding

GO: 0003682: chromatin binding

GO: 0005178: integrin binding

GO: 0000217: DNA secondary structure binding

GO: 0043515: kinetochore binding

GO: 0016301: kinase activity

GO: 0050840: extracellular matrix binding

GO: 0005518: collagen binding

GO: 0050662: coenzyme binding

GO: 0050211: procollagen galactosyltransferase activity GO: 0000287: magnesium ion binding

G: 0000775: chromosome, centromeric region GO: 0005819: spindle

GO: 0005815: microtubule organizing center

GO: 0000794: condensed nuclear chromosome

GO: 0051233: spindle midzone

GO: 0005788: endoplasmic reticulum lumen

GO: 0031298: replication fork protection complex

GO: 0032133: chromosome passenger complex

GO: 0005925: focal adhesion

GO: 0005583: fibrillar collagen trimer

GO: 0031262: Ndc80 complex

GO: 0000796: condensin complex

GO: 0045120: pronucleus

GO: 0042599: lamellar body

GO: 0000781: chromosome, telomeric region

GO: 0044450: microtubule organizing center part

GO: 0097134: cyclin E1-CDK2 complex

GO: 0097149: centralspindlin complex

GO: 0032432: actin filament bundle

GO: 0005680: anaphase-promoting complex 


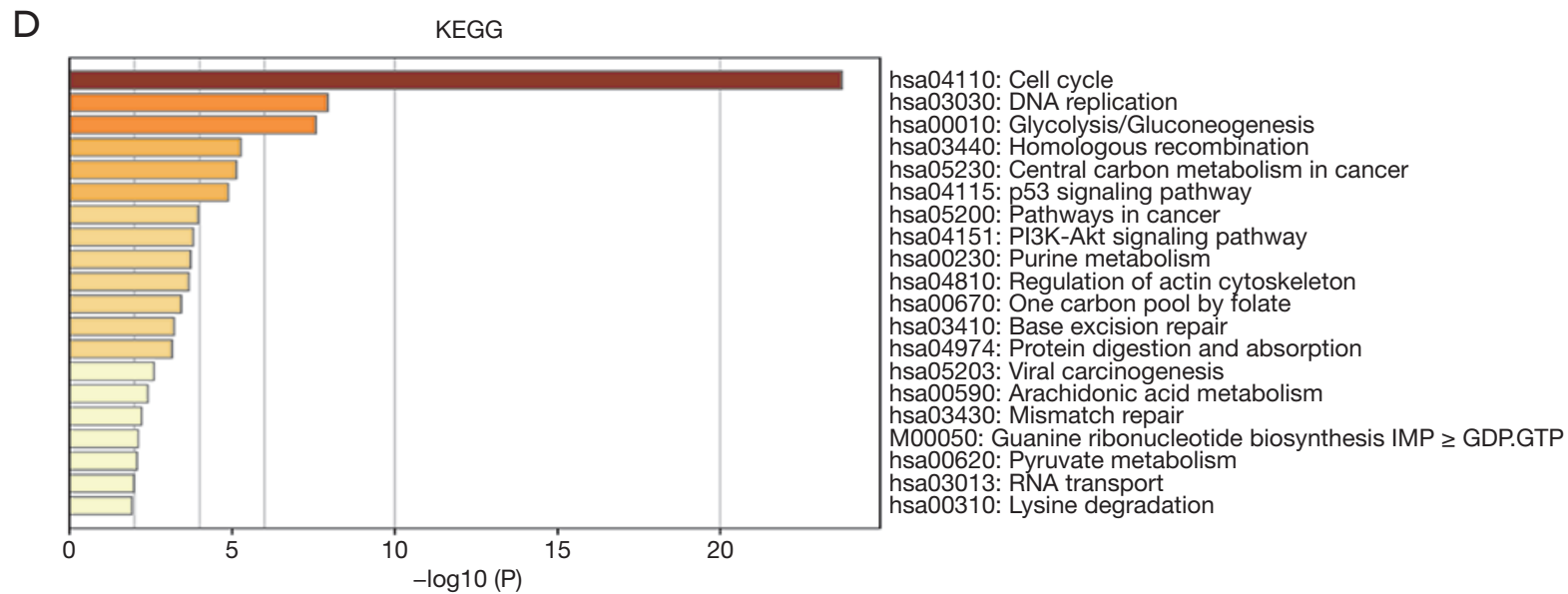

Figure 8 Functional enrichment analysis of the coexpressed genes of PLODs in LUAD (Metascape). (A,B,C) GO enrichment analysis, including BP, CC, and MF. (D) KEGG enrichment analysis. PLOD, procollagen-lysine,2-oxoglutarate 5-dioxygenase; LUAD, lung adenocarcinoma; GO, Gene Ontology; BP, biological processes; CC, cellular components; MF, molecular functions; KEGG, Kyoto Encyclopedia of Genes.

crosslinks $(31,32)$. Increasing evidence has shown that the overexpression of PLOD2 facilitates cancer progression $(16,17,29)$. Du et al. (16) reported that the high expression of PLOD2 significantly enhanced the migration of lung cancer cells in vitro, while the knockdown of PLOD2 weakened the metastasis of lung cancer cells in vivo in a mouse model. Du et al. (16) also investigated the high expression of PLOD2 was significantly relevant to poor prognosis in patients with LUAD but not in patients with LUSC. We found similar prognostic results for PLOD2 in LUAD and LUSC. Similar to the function of PLOD2 in lung cancer, PLOD3 plays an important role in the metastasis of lung cancer (28). In addition, PLOD3 participates in the therapeutic effect of lung cancer (33). Baek and colleagues have shown that the knockdown of PLOD3 decreases chemoresistance and radioresistance by promoting apoptosis in lung cancer (33).

In our report, the enrichment analysis of the KEGG pathway showed that the functions of PLOD family members focus on the cell cycle, DNA replication, and glycolysis/gluconeogenesis in LUAD. PLOD family members also participate in the $\mathrm{p} 53$ signaling pathway. Yamada et al. (30) reported that the proportion of cells in the G0/G1 phase in bladder cancer cells treated with a PLOD1 inhibitor was significantly higher than that in the control cells. The same result was observed in bladder cancer cells with knockdown of PLOD1 in this study (30). DNA replication is a vulnerable biological process and can cause genomic instability, which leads to the initiation and progression of cancer (34). A pathway analysis indicated that PLODs also participate in the $\mathrm{p} 53$ signaling pathway. Previous studies have shown that the p53 signaling pathway has a key role in multiple cellular processes, including the cell cycle and cancer progression $(35,36)$. TP53 can encode the $\mathrm{p} 53$ protein and is identified as a tumor suppressor gene with a high-frequency mutation in cancer. Wild-type TP53 can weaken tumor progression, whereas TP53 mutations can strengthen tumor development and facilitate the escape from tumor cell death (37). In our study, we found that the mRNA expression levels of PLODs from tumor tissues with the TP53 mutation were significantly higher than those without the TP53 mutation in LUAD but not in LUSC. In recent years, the important role of metabolism has attracted increasing attention in cancer. Several studies have demonstrated that glycolysis significantly influences the antitumor immunity of $\mathrm{T}$ cells and tumor growth $(38,39)$. In the future, the association between PLODs and glycolysis should be further explored in LUAD. Compared with LUAD, the pathway analysis of LUSC revealed that PLODs were involved in ECM-receptor interaction, protein processing in the endoplasmic reticulum, and glycosaminoglycan biosynthesis. The enrichment analysis of GO also indicates that PLODs focus on the ECM. Numerous studies have demonstrated that the ECM plays a critical role in cancer progression (3-5). The formation of collagen, the most abundant component of the ECM, is 
regulated by PLOD family members. Increasing evidence has demonstrated that abnormal collagen deposition and crosslinking can participate in malignant biological processes, including proliferation, invasion, and migration (40-42).

Evading immune surveillance and attack is an important hallmark of cancer (43). Recently, immunotherapies targeting immune checkpoint proteins, including programmed cell death 1 and PD-L1, have shown remarkable clinical efficacy in various cancers (44-46). Nevertheless, a majority of patients with cancer are not sensitive to immune checkpoint blockade. The molecular profiles clarifying the resistance of immune checkpoint blockade have yet to be comprehensively investigated. In our report, the results show that the expression levels of PLOD1/2/3 have significant relevance to the infiltrating levels of some immune-related cells. PLODs are also associated with several immune-related genes, including CD274, CXCL10, GZMA, and GZMB. CXCL10, $G Z M A$, and $G Z M B$ are considered as key genes related to activated $\mathrm{T}$ cells and immune cytolytic activity $(47,48)$. Further analysis showed that the expression of PLOD2 had the most evident correlations with immune-related genes in LUAD. To the best of our knowledge, the correlation between PLOD2 and tumor immunity has not been investigated. In the future, the effect of PLOD2 on tumor immunity should be deeply explored.

Some limitations were observed in our study. First, data analyses are mainly performed in online databases. The number of clinical samples with LUAD and LUSC was small, and the difference in survival could not be accurately evaluated. Thus, our results should be confirmed by further study based on abundant clinical sample data. Second, because the data are derived from public databases, some clinical characteristics were lacking and could not be obtained to improve the statistical reliability and validity. Finally, the potential molecular mechanisms of PLOD family members in lung cancer were not investigated.

\section{Conclusions}

In conclusion, our study demonstrated that the mRNA and protein expression levels of PLOD1/2/3 were up-regulated in LUAD and LUSC tissues. High expression levels of PLOD $1 / 2 / 3$ were significantly related to poor OS and PFS in LUAD but not in LUSC. In addition, PLOD1 and PLOD3 could serve as independent risk factors in LUAD. We also observed a high alteration rate of PLODs in patients with LUSC and the genetic alterations of PLODs had significant relevance to favorable OS in these patients. Furthermore, we found that PLOD family members were significantly associated with immune-infiltrating cells and immune-related genes in LUAD and LUSC. Interestingly, compared with PLOD1/3 expression, PLOD2 expression in LUAD had the most evident correlations with immunerelated genes (i.e., CD274, CXCL10, GZMA, and GZMB). Therefore, PLODs may be used as suitable prognostic markers in the future.

\section{Acknowledgments}

Funding: None.

\section{Footnote}

Reporting Checklist: The authors have completed the REMARK reporting checklist. Available at https://dx.doi. org/10.21037/tcr-21-73

Data Sharing Statement: available at https://dx.doi. org/10.21037/tcr-21-73

Conflicts of Interest: All authors have completed the ICMJE uniform disclosure form (available at https://dx.doi. org/10.21037/tcr-21-73). The authors have no conflicts of interest to declare.

Ethical Statement: The authors are accountable for all aspects of the work in ensuring that questions related to the accuracy or integrity of any part of the work are appropriately investigated and resolved. The study was conducted in accordance with the Declaration of Helsinki (as revised in 2013). The study was approved by the institutional review board of Tianjin Medical University Cancer Institute and Hospital (NO.: bc2020193) and informed consent was taken from all the patients.

Open Access Statement: This is an Open Access article distributed in accordance with the Creative Commons Attribution-NonCommercial-NoDerivs 4.0 International License (CC BY-NC-ND 4.0), which permits the noncommercial replication and distribution of the article with the strict proviso that no changes or edits are made and the original work is properly cited (including links to both the formal publication through the relevant DOI and the license). See: https://creativecommons.org/licenses/by-nc-nd/4.0/. 


\section{References}

1. Bray F, Ferlay J, Soerjomataram I, et al. Global cancer statistics 2018: GLOBOCAN estimates of incidence and mortality worldwide for 36 cancers in 185 countries. CA Cancer J Clin 2018;68:394-424.

2. Siegel RL, Miller KD, Jemal A. Cancer statistics, 2018. CA Cancer J Clin 2018;68:7-30.

3. Gilkes DM, Semenza GL, Wirtz D. Hypoxia and the extracellular matrix: drivers of tumour metastasis. Nat Rev Cancer 2014;14:430-9.

4. Götte M, Kovalszky I. Extracellular matrix functions in lung cancer. Matrix Biol 2018;73:105-21.

5. Kaushik N, Kim S, Suh Y, et al. Proinvasive extracellular matrix remodeling for tumor progression. Arch Pharm Res 2019;42:40-7.

6. Chen Y, Guo H, Terajima M, et al. Lysyl Hydroxylase 2 Is Secreted by Tumor Cells and Can Modify Collagen in the Extracellular Space. J Biol Chem 2016;291:25799-808.

7. Egeblad M, Rasch MG, Weaver VM. Dynamic interplay between the collagen scaffold and tumor evolution. Curr Opin Cell Biol 2010;22:697-706.

8. Barnes MJ, Constable BJ, Morton LF, et al. Age-related variations in hydroxylation of lysine and proline in collagen. Biochem J 1974;139:461-8.

9. Valtavaara M, Szpirer C, Szpirer J, et al. Primary structure, tissue distribution, and chromosomal localization of a novel isoform of lysyl hydroxylase (lysyl hydroxylase 3). J Biol Chem 1998;273:12881-6.

10. Gjaltema RA, Bank RA. Molecular insights into prolyl and lysyl hydroxylation of fibrillar collagens in health and disease. Crit Rev Biochem Mol Biol 2017;52:74-95.

11. Szpirer C, Szpirer J, Rivière M, et al. Localization of the gene encoding a novel isoform of lysyl hydroxylase. Mamm Genome 1997;8:707-8.

12. Tasker PN, Macdonald H, Fraser WD, et al. Association of PLOD1 polymorphisms with bone mineral density in a population-based study of women from the UK. Osteoporos Int 2006;17:1078-85.

13. Chen ZQ, Huang LS, Zhu B. Assessment of Seven Clinical Tumor Markers in Diagnosis of Non-Small-Cell Lung Cancer. Dis Markers 2018;2018:9845123.

14. Wang D, Zhang S, Chen F. High Expression of PLOD1 Drives Tumorigenesis and Affects Clinical Outcome in Gastrointestinal Carcinoma. Genet Test Mol Biomarkers 2018;22:366-73.

15. Li SS, Lian YF, Huang YL, et al. Overexpressing PLOD family genes predict poor prognosis in gastric cancer. J
Cancer 2020;11:121-31.

16. Du H, Chen Y, Hou X, et al. PLOD2 regulated by transcription factor FOXA1 promotes metastasis in NSCLC. Cell Death Dis 2017;8:e3143.

17. Song Y, Zheng S, Wang J, et al. Hypoxia-induced PLOD2 promotes proliferation, migration and invasion via $\mathrm{PI} 3 \mathrm{~K} /$ Akt signaling in glioma. Oncotarget 2017;8:41947-62.

18. Chandrashekar DS, Bashel B, Balasubramanya SAH, et al. UALCAN: A Portal for Facilitating Tumor Subgroup Gene Expression and Survival Analyses. Neoplasia 2017;19:649-58.

19. Uhlén M, Fagerberg L, Hallström BM, et al. Proteomics. Tissue-based map of the human proteome. Science 2015;347:1260419.

20. Győrffy B, Surowiak P, Budczies J, et al. Online survival analysis software to assess the prognostic value of biomarkers using transcriptomic data in non-small-cell lung cancer. PLoS One 2013;8:e82241.

21. Cerami E, Gao J, Dogrusoz U, et al. The cBio cancer genomics portal: an open platform for exploring multidimensional cancer genomics data. Cancer Discov 2012;2:401-4.

22. Zhou Y, Zhou B, Pache L, et al. Metascape provides a biologist-oriented resource for the analysis of systemslevel datasets. Nat Commun 2019;10:1523.

23. Li T, Fan J, Wang B, et al. TIMER: A Web Server for Comprehensive Analysis of Tumor-Infiltrating Immune Cells. Cancer Res 2017;77:e108-10.

24. Garber ME, Troyanskaya OG, Schluens K, et al. Diversity of gene expression in adenocarcinoma of the lung. Proc Natl Acad Sci U S A 2001;98:13784-9.

25. Hou J, Aerts J, den Hamer B, et al. Gene expressionbased classification of non-small cell lung carcinomas and survival prediction. PLoS One 2010;5:e10312.

26. Landi MT, Dracheva T, Rotunno M, et al. Gene expression signature of cigarette smoking and its role in lung adenocarcinoma development and survival. PLoS One 2008;3:e1651.

27. Talbot SG, Estilo C, Maghami E, et al. Gene expression profiling allows distinction between primary and metastatic squamous cell carcinomas in the lung. Cancer Res 2005;65:3063-71.

28. Baek JH, Yun HS, Kwon GT, et al. PLOD3 promotes lung metastasis via regulation of STAT3. Cell Death Dis 2018;9:1138.

29. Xu F, Zhang J, Hu G, et al. Hypoxia and TGF- $\beta 1$ induced PLOD2 expression improve the migration and invasion of cervical cancer cells by promoting epithelial- 
to-mesenchymal transition (EMT) and focal adhesion formation. Cancer Cell Int 2017;17:54.

30. Yamada Y, Kato M, Arai T, et al. Aberrantly expressed PLOD1 promotes cancer aggressiveness in bladder cancer: a potential prognostic marker and therapeutic target. Mol Oncol 2019;13:1898-912.

31. Gilkes DM, Bajpai S, Chaturvedi P, et al. Hypoxiainducible factor 1 (HIF-1) promotes extracellular matrix remodeling under hypoxic conditions by inducing $\mathrm{P} 4 \mathrm{HA} 1$, P4HA2, and PLOD2 expression in fibroblasts. J Biol Chem 2013;288:10819-29.

32. Yamauchi M, Sricholpech M. Lysine post-translational modifications of collagen. Essays Biochem 2012;52:113-33.

33. Baek JH, Yun HS, Kwon GT, et al. PLOD3 suppression exerts an anti-tumor effect on human lung cancer cells by modulating the PKC-delta signaling pathway. Cell Death Dis 2019;10:156.

34. Gaillard H, García-Muse T, Aguilera A. Replication stress and cancer. Nat Rev Cancer 2015;15:276-89.

35. Kruiswijk F, Labuschagne CF, Vousden KH. p53 in survival, death and metabolic health: a lifeguard with a licence to kill. Nat Rev Mol Cell Biol 2015;16:393-405.

36. Tanikawa C, Zhang YZ, Yamamoto R, et al. The Transcriptional Landscape of p53 Signalling Pathway. EBioMedicine 2017;20:109-19.

37. Bykov VJN, Eriksson SE, Bianchi J, et al. Targeting mutant p53 for efficient cancer therapy. Nat Rev Cancer 2018;18:89-102.

38. Cascone T, McKenzie JA, Mbofung RM, et al. Increased Tumor Glycolysis Characterizes Immune Resistance to Adoptive T Cell Therapy. Cell Metab 2018;27:977-87.e4.
39. Zeng C, Wu Q, Wang J, et al. NOX4 supports glycolysis and promotes glutamine metabolism in non-small cell lung cancer cells. Free Radic Biol Med 2016;101:236-48.

40. Zhu J, Xiong G, Fu H, et al. Chaperone Hsp47 Drives Malignant Growth and Invasion by Modulating an ECM Gene Network. Cancer Res 2015;75:1580-91.

41. Provenzano PP, Eliceiri KW, Campbell JM, et al. Collagen reorganization at the tumor-stromal interface facilitates local invasion. BMC Med 2006;4:38.

42. Provenzano PP, Inman DR, Eliceiri KW, et al. Collagen density promotes mammary tumor initiation and progression. BMC Med 2008;6:11.

43. Hanahan D, Weinberg RA. Hallmarks of cancer: the next generation. Cell 2011;144:646-74.

44. Cohen EEW, Soulières D, Le Tourneau C, et al. Pembrolizumab versus methotrexate, docetaxel, or cetuximab for recurrent or metastatic head-and-neck squamous cell carcinoma (KEYNOTE-040): a randomised, open-label, phase 3 study. Lancet 2019;393:156-67.

45. Hamid O, Robert C, Daud A, et al. Safety and tumor responses with lambrolizumab (anti-PD-1) in melanoma. N Engl J Med 2013;369:134-44.

46. Brahmer J, Reckamp KL, Baas P, et al. Nivolumab versus Docetaxel in Advanced Squamous-Cell Non-Small-Cell Lung Cancer. N Engl J Med 2015;373:123-35.

47. Rooney MS, Shukla SA, Wu CJ, et al. Molecular and genetic properties of tumors associated with local immune cytolytic activity. Cell 2015;160:48-61.

48. Tuo Z, Zong Y, Li J, et al. PD-L1 regulation by SDH5 via $\beta$-catenin/ZEB1 signaling. Oncoimmunology 2019;8:1655361.
Cite this article as: Qi Q, Huang W, Zhang H, Zhang B, Sun $\mathrm{X}$, Ma J, Zhu C, Wang C. Bioinformatic analysis of PLOD family member expression and prognostic value in non-small cell lung cancer. Transl Cancer Res 2021;10(6):2707-2724. doi: $10.21037 /$ tcr-21-73 
A
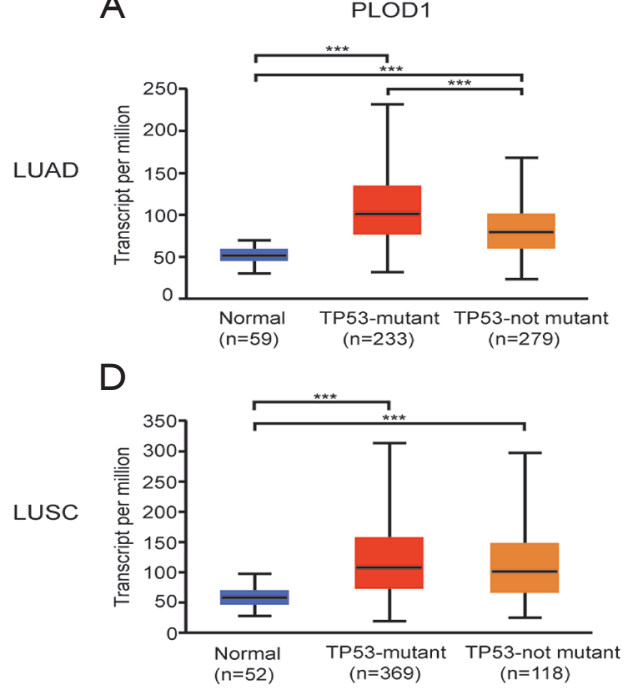

B

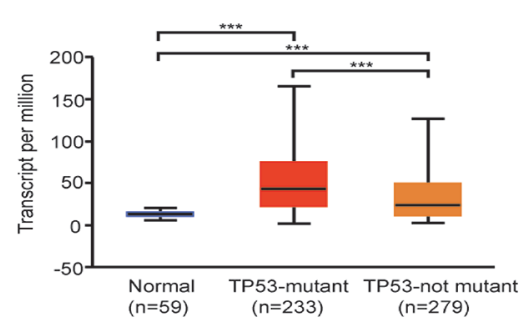

E

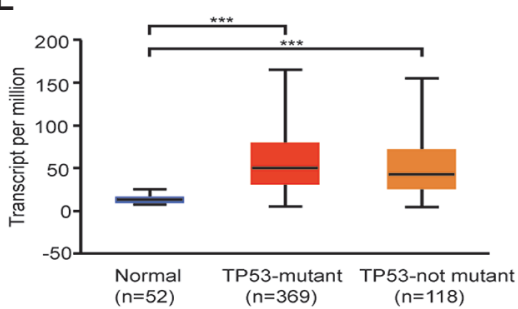

C

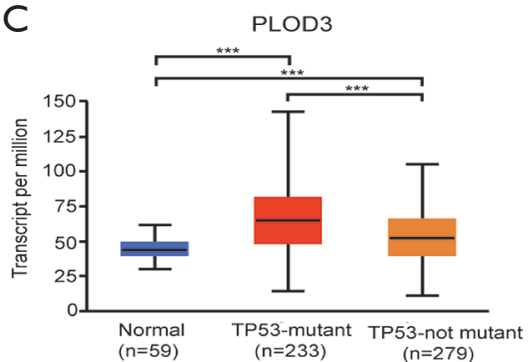

$\mathrm{F}$

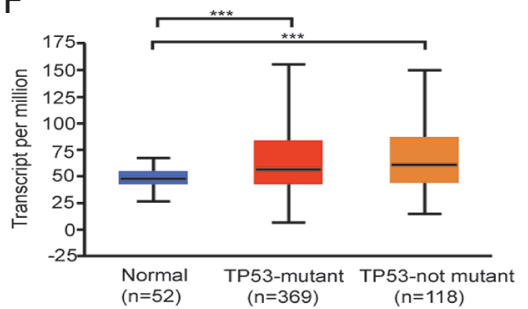

Figure S1 Correlation between the mRNA expression of PLODs and TP53 mutation in patients with lung cancer (UALCAN). Correlation between PLOD expression and TP53 mutation in (A,B,C) LUAD and (D,E,F) LUSC. ${ }^{* * *}, \mathrm{P}<0.001$. PLOD, procollagen-lysine, 2-oxoglutarate 5-dioxygenase; LUAD, lung adenocarcinoma; LUSC, lung squamous cell carcinoma.

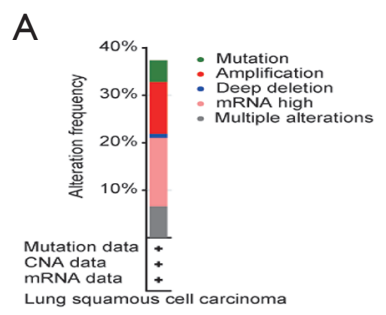

C

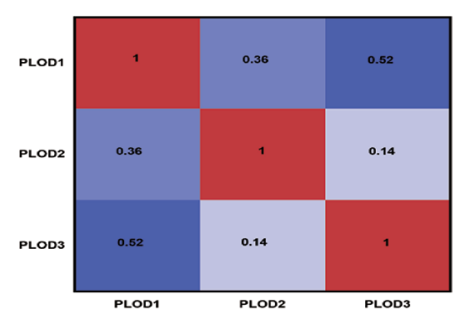

\section{B}

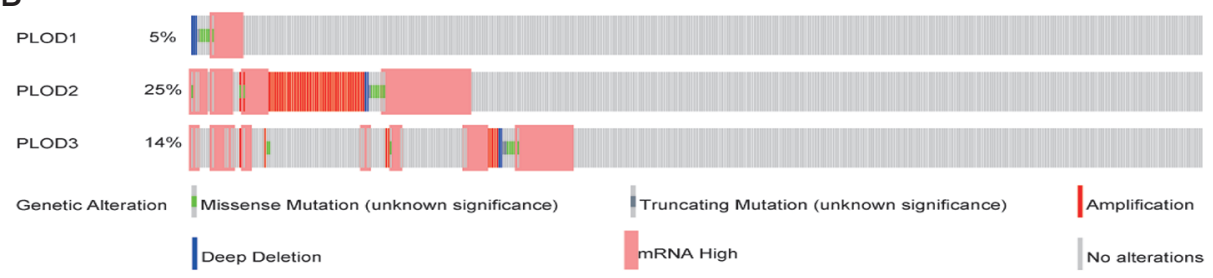

D

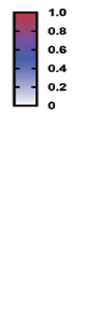

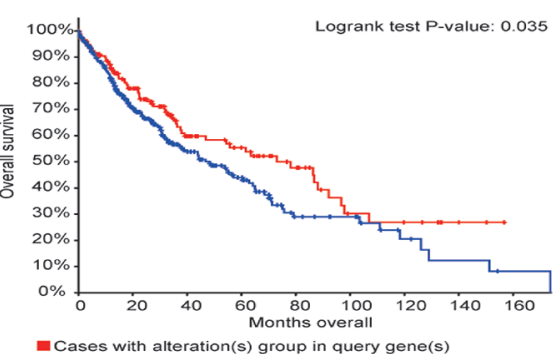

ECases with alteration(s) group in query gene(s)
Cases without alteration(s) group in query gene(s)
$\mathrm{E}$

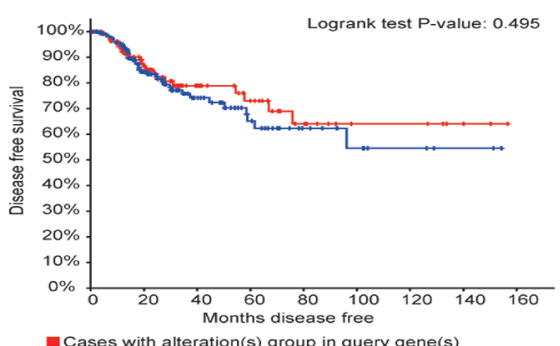

- Cases with alteration(s) group in query gene(s)
Cases without alteration(s) group in query gene(s)

Figure S2 Expression mutation analysis of PLODs in LUSC (cBioPortal). (A) Summary of genetic alterations in PLODs. (B) Genetic alterations in individual PLOD family members. (C) Pearson correlation among PLOD1, PLOD2, and PLOD3. (D,E) Correlation between genetic alterations and survival (OS and DFS). PLOD, procollagen-lysine,2-oxoglutarate 5-dioxygenase; LUSC, lung squamous cell carcinoma; OS, overall survival; DFS, disease-free survival. 


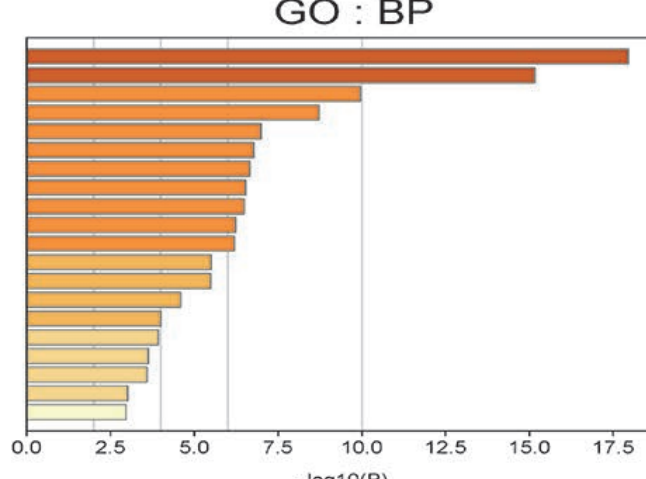

C

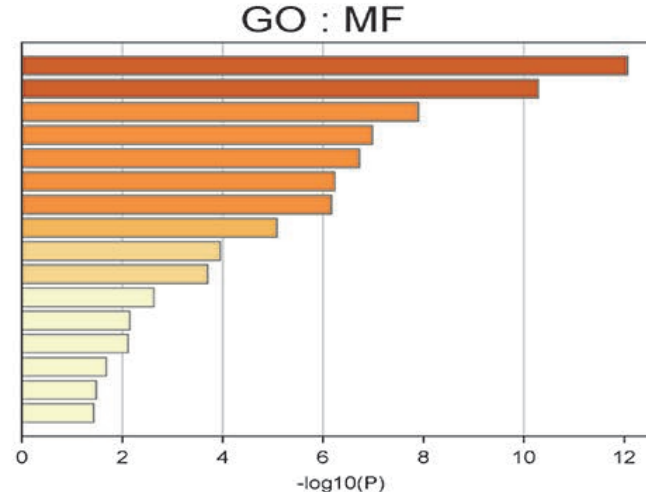

B
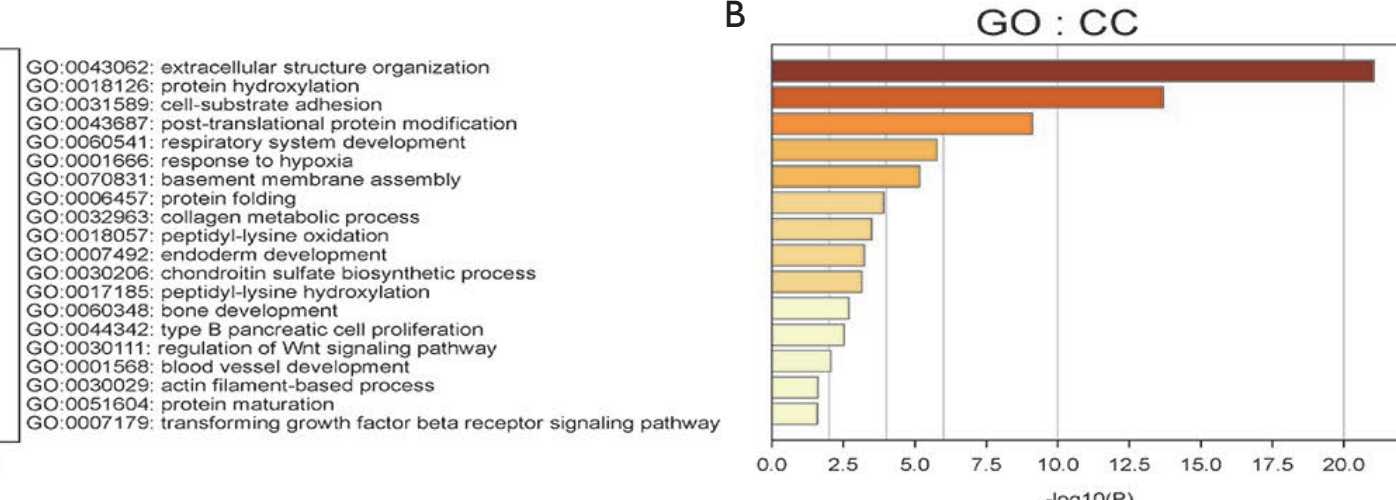

GO:0031418: L-ascorbic acid binding O:0031418: L-ascorbic acid binding
$0: 0005201:$ extracellular matrix structural constituent
$0: 0015036$ : disulfide oxidoreductase activity o.:0055509: calcium ion binding O:0050839: cell adhesion molecule binding .0.005518. collagen binding (a) GO 0051087 : chaperone binding GO:0051020: GTPase binding
GO:0044389: ubiquitin-like protein ligase binding
GO:0042277: peptide binding :0042277: peptide binding

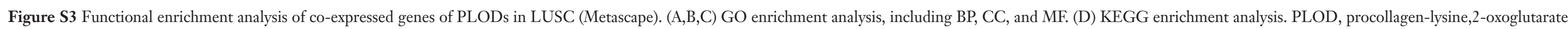
5-dioxygenase. GO, Gene Ontology; BP, biological processes; CC, cellular components; MF, molecular functions; KEGG, Kyoto Encyclopedia of Genes.
GO:0005788: endoplasmic reticulum lumen

GO:0031012: extracellular matrix

GO:0032580: Golgi cisterna membrane

G:0034663: endoplasmic reticulum chaperone complex

GO:0098552: side of membrane

(00.0048471:

Go: 0005796: Golgi lumen

GO:0031300: intrinsic component of organelle membrane

GO:0031252: cell leading edge

GO:0019897: extrinsic component of plasma membrane

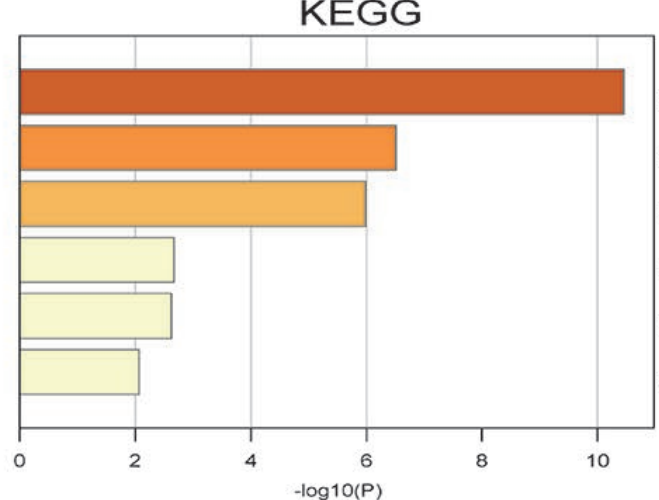

hsa04512: ECM-receptor interaction

hsa04141: Protein processing in endoplasmic reticulum

hsa00532: Glycosaminoglycan biosynthesis - chondroitin sulfate / dermatan sulfate

hsa05131: Shigellosis

hsa00010: Glycolysis / Gluconeogenesis

hsa05205: Proteoglycans in cancer 


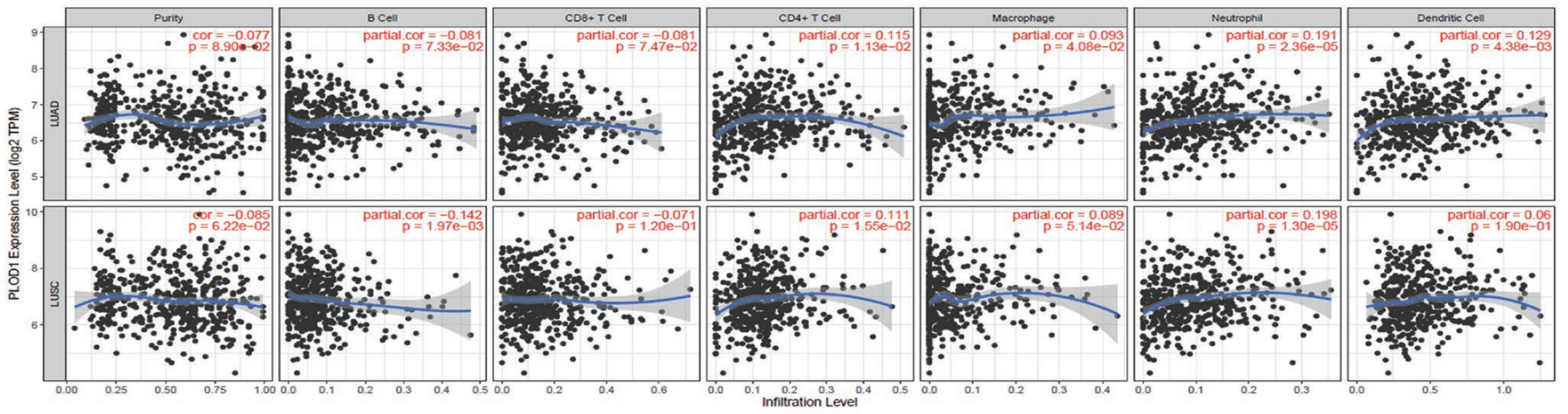

B
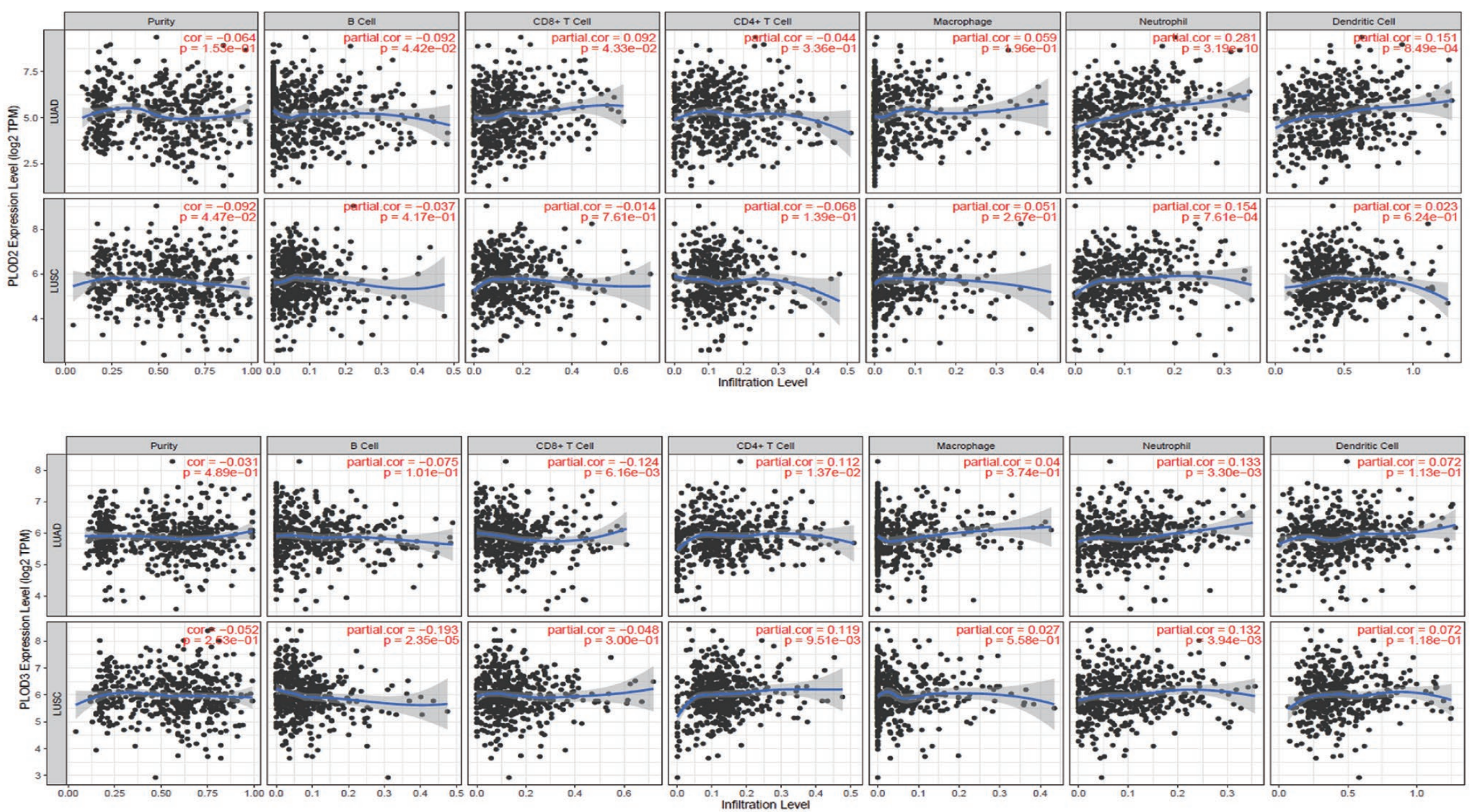

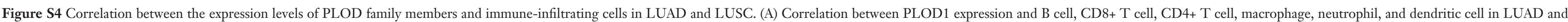

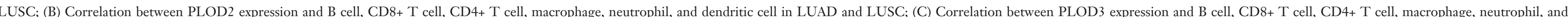
dendritic cell in LUAD and LUSC. PLOD, procollagen-lysine,2-oxoglutarate 5-dioxygenase; LUAD, lung adenocarcinoma; LUSC, lung squamous cell carcinoma. 

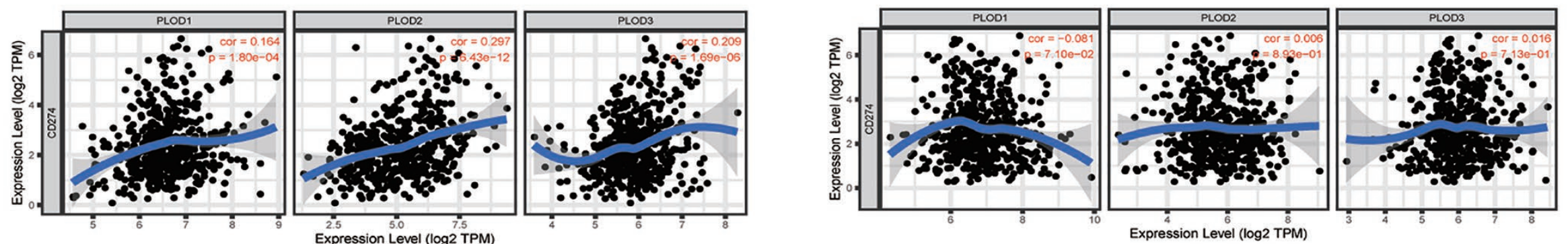

C
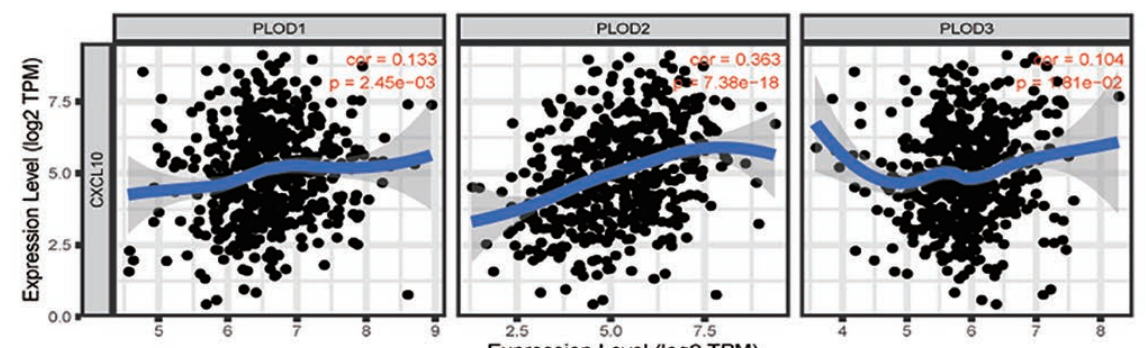

D
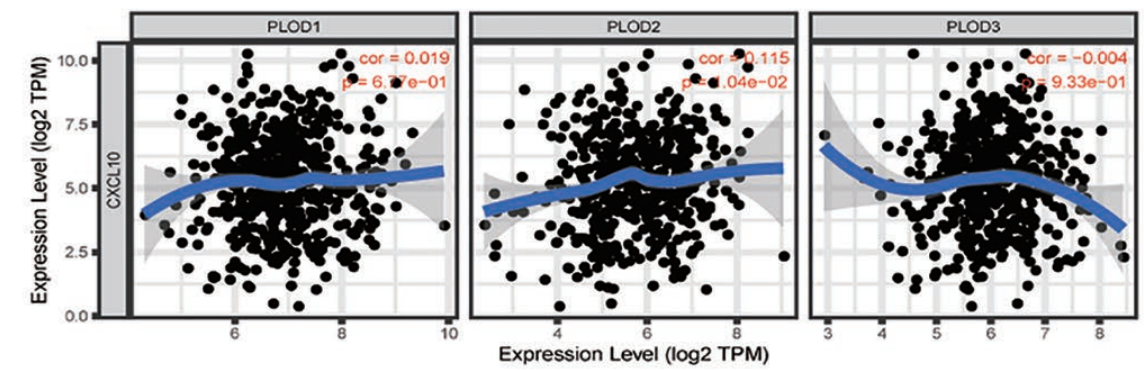

E
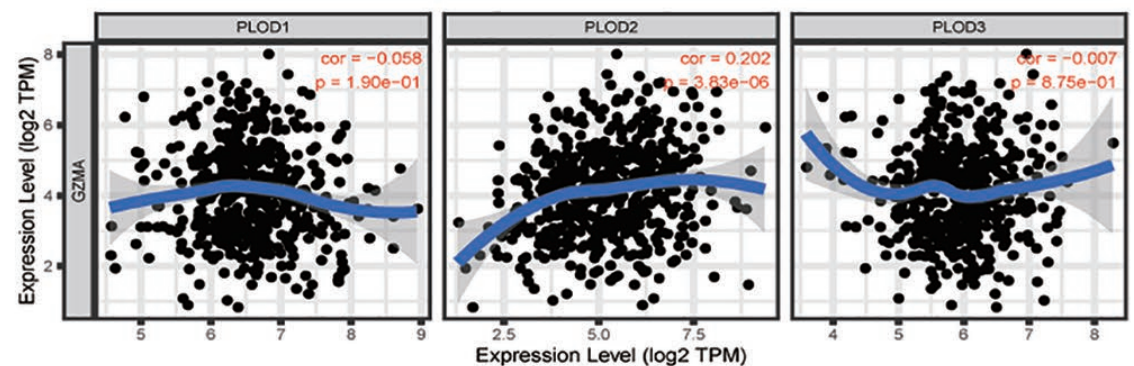

F
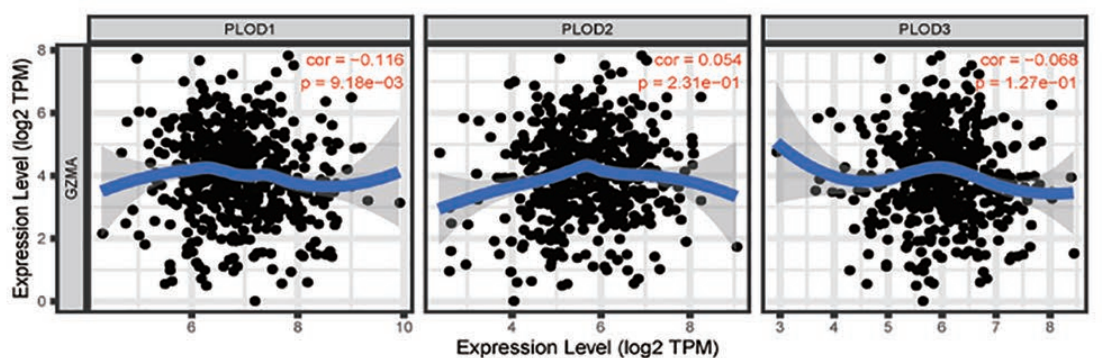

G
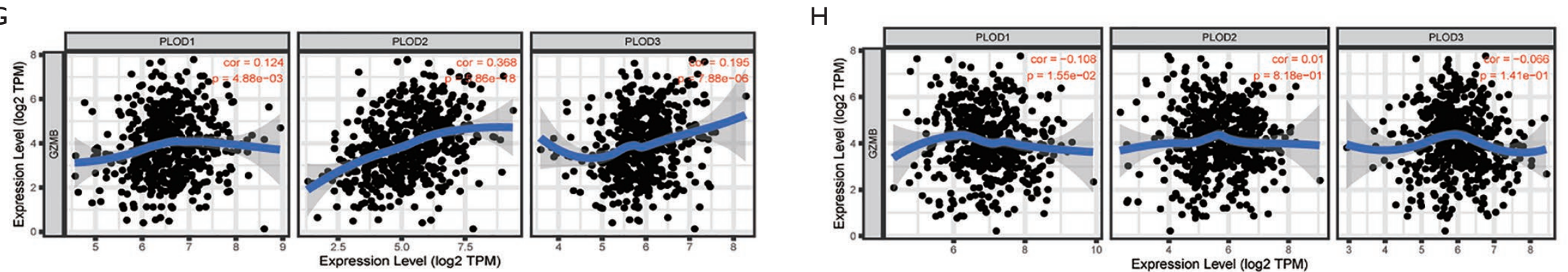

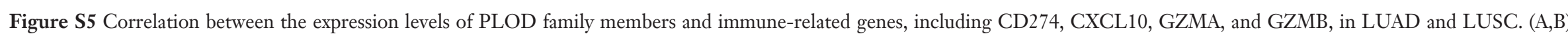

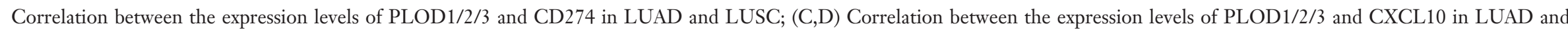

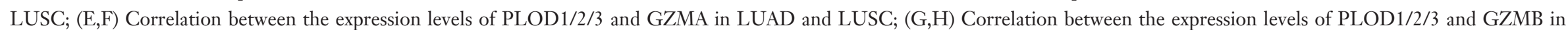

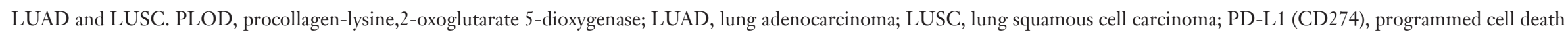
1 ligand 1; CXCL10, C-X-C motif chemokine ligand 10; GZMA, granzyme A; GZMB, granzyme B. 